\title{
Phytoextraction and translocation of cadmium in saline soil by hemerocallis fulva and dodonaea viscosa plants
}

\begin{abstract}
Purpose: Cadmium contaminated Saline soil has always been a problem for sustainable agriculture and environment. Cadmium $(\mathrm{Cd})$ is a noxious heavy metal and its co-occurrence with high salt $(\mathrm{NaCl})$ concentrations in soil decreases quality of food and quantity of crops. For this purposes to search the salinity problems in $\mathrm{Cd}$ contaminated soil, its uptake and accumulation and its effect on plant growth and biomass were studied in two terrestrial plants.
\end{abstract}

Materials and methods: Different concentration of salt, $\mathrm{NaCl}(1000,3000$ and $6000 \mathrm{ppm})$ in combination with $\mathrm{Cd}$ metal $(50,100$ and $150 \mathrm{ppm})$ were added into pots soil, the two plants (Hemerocallis fulva and Dodonaea viscosa) were grown in it. For control (C) is used having no cadmium and salt while the remaining three with diverse concentrations of $\mathrm{Cd}(\mathrm{C} 1=50 \mathrm{ppm}, \mathrm{C} 2=100 \mathrm{ppm}$ and $\mathrm{C} 3=150 \mathrm{ppm})$.

Results and Discussion: Plant biomass and growth were highly reduced under variable concentrations of $\mathrm{Cd}$ and salt in soil. Combination of $6000 \mathrm{ppm} \mathrm{NaCl}$ and $150 \mathrm{ppm}$ $\mathrm{Cd}$ in soil demonstrated highest significant $\mathrm{Cd}$ accumulation in the plants. Dodonaea viscosa showed high Cd-bio concentration value (more than one) as compared to Hemerocallis fulva having less than one. It was noted that Dodonaea viscosa plant accumulates maximum concentration of $\mathrm{Cd}$ in sodium salt than Hemerocallis fulva plant.

Conclusion: Dodoneae plant potentially hyper accumulator and showed enough tolerance to high concentration of salt during Phytoextraction of $\mathrm{Cd}$. It is strongly recommended that such plants should be planted in metal contaminated saline soil and also for the conservation of barren soil.

Keywords: terrestrial plants, salinity, soil, phytoextraction
Volume 7 Issue 5 - 2017

\author{
Ayaz Ahmad,' Fazal Hadi, ${ }^{2}$ Nasir Ali ${ }^{3}$ \\ 'Department of Biological Sciences, Swat College of Science and \\ Technology Swat, Pakistan \\ ${ }^{2}$ Department of Biotechnology, University of Malakand, \\ Chakdara, Pakistan \\ ${ }^{3}$ Department of Biotechnology, Sarhad University Peshawar, \\ Pakistan
}

Correspondence: Ayaz Ahmad, Department of Biological Sciences, Swat College of Science and Technology Swat, KhyberPakhtunkhwa, Pakistan, Email qariayaz@gmail.com

Received: May 04, 2017 | Published: October 10, 2017

\section{Introduction}

Availability of clean and fresh water is a global issue, environmental threat, life span indicator, remedy to cell metabolism and physiological activities are totally depend upon the fresh water Globally high salt concentration in the soil is a major issue for arid, semi-arid and oceanic region in the world. ${ }^{1,2}$ It generates responses in plants, changing in oxidation reduction processes in the cell, plant yield and growth as well as biomass ${ }^{3,4}$ Such environmental stress conditions influence the root relation with surrounding and ascent poisonous materials within the plants. World widely $955 \mathrm{M}$ ha, soil is primary affected by salt while increasing rate of salinity secondary affect $77 \mathrm{M}$ ha, in which $58 \%$ is through irrigation. In Asia there is approximately 21.5 million hectare soil is salt affected by major ions like $\mathrm{Na}^{+}, \mathrm{K}^{+}, \mathrm{Ca}^{++}, \mathrm{Mg}^{++}$and $\mathrm{Cl}^{-}$. Hyper salinization severely impact on crops to reduce growth, yields and productivity because it is a vital environmental stress. ${ }^{5}$ Salt concentrations also influence seedling, cellular plant metabolism due to osmotic stress, oxidative stress and particular effects of ions. ${ }^{6}$

From the collected data revealed that some of the plants are very tolerance to salinity specially Barley, chick pea. ${ }^{7}$ In Pakistan, majority places in plane areas containing maximum amount of salt in soil than needed, such soil is physiologically draught and barren while worthless for cultivation of crops, vegetables and other plants and also produced environmental issue for the people and countries. Other than some soils also having toxic metals which further make soil noxious for cultivation and crops purposes. Salt stress is an environmental issue for control process of limited resource of plant growth, production, distribution and abundance of a population. In recent survey report estimated that majority of irrigation water is either alkaline or saline about one third of irrigation section ${ }^{8}$ Cadmium circulation rate is high and easily be absorbed and translocated by plants because its low soil holding capacity. ${ }^{9}$ Cadmium is so toxic when enters into living through food chain can generate chronic health problems. ${ }^{10}$

The regulatory limit of cadmium (Cd) in agricultural soil is $100 \mathrm{mg} /$ $\mathrm{kg}$ soil, ${ }^{11}$ but this threshold is continuously exceeding because of several human activities. Plants exposed to high levels of $\mathrm{Cd}$ causes reduction in photosynthesis, water uptake, and nutrient uptake. Hypertonic salt solution in soil decrease germination of seeds, seedling, flowering and fruit formation, quantity and quality of crops. ${ }^{12}$ Maximum amount or above the normal amount of salt develops osmotic shock disturbs ionic concentration in plant cells, ${ }^{13}$ which ultimately denature physiologically activities of plants on cellular level as well as on the whole plants by osmotic and ionic stress. Dissolved salts make isotonic environment for crops, but some plants are tolerate to salt, metals and enable to absorb maximum amount of metals along with salts, simply to absorb maximum amount of metals along with salts, from naturally saline substrates like bio solids (organic nutrients 
prepared from the treatment of manure ,thick, soft and wet mud as well as industrial or refinery sludge. According to the report of, ${ }^{14}$ Plants adopt their selves with water stress and salt stress condition through osmosis by intake or lose of substances to maintain their status due to change the internal photosynthetic enzymatic quantity and balance because of external shade and light stress (helio and hele stress). By facing these problems, Biologists compelled to search solution for the homeostasis of soil contents, purification of water and cleaning of environment. Due to biological researchers, a green technology aroused in the shape of phyto remediation, that using of variety hyper accumulator plants to reduce the level of salinity and metals in the polluted and affected soil. The plants having the ability to continue their physiological activities under salt stress condition are suggested for exploiting tolerant cultivars of coastal area, because salt minimize plant growth, alter nutritional balance, specific chemical ionic reactions and homeostasis of plants, ${ }^{15}$ Which destroy the nature of membrane and produce activated oxygen and cause metal toxicity. Salinity is a vast field of abiotic stress that restrict plant growth and its ecological distribution all over the world especially in Pakistan and Egypt ${ }^{16,17}$ and ${ }^{18}$ Cellular metabolisms like growth, photosynthesis, protein and lipid metabolism of plant are severely affected by hyper penetration of soluble salts in arid and semiarid soil region. ${ }^{19}$ Salt stress can affect growth in plants because of osmotic effect of salt is closely identical to water stress of drought. Secondly when the salt enters to plant causing toxicity in older transpired leaves and ultimately shows premature death. For phyto remediation, Hemerocallis and Dodneae plants having good potentiality to extract and translocase salts and metals from enriched saline metal soils into harvestable parts.

\section{Materials and methods}

\section{Media (Soil) preparation and plants Transformation}

Soil was collected from the herbarium of the University of Malakand, dried in sun light then grinded into fine powdered form and poured into clay pots ( $3 \mathrm{~kg}$ soil $/$ pot). Water holding capacity $(250 \mathrm{~mL}$ water $/ \mathrm{kg}$ soil \pm 4$)$, electrical conductivity $(814 \mu \mathrm{s} \pm 7)$ and $\mathrm{pH}$ $(6.7 \pm 2)$ of the soil was measured. Two different plants (Dodonaea viscosa and Hemerocallis fulva,) were used during the experiment. After germination uniform size plantlets $(2 \mathrm{~cm}$ roots and $3 \mathrm{~cm}$ shoot) were selected for the experiment.

\section{Treatments given to plants during the experiment}

In the whole experiment Cadmium acetate dehydrate $(\mathrm{Cd})$ solution were added to plants in three different concentrations $(50,100$ and $150 \mathrm{ppm})$ along with sodium chloride salt in the manner of three different concentrations (1000, 3000 and 6000ppm). Whole experiment was carried out in complete randomised design (CRD) in the manner of three replicates and one control under natural light/dark conditions with temperature $30 / 250 \mathrm{C}$. The following treatments and control (Table 1) were used during the experiment.

\section{Measurement of plant's Parameters}

To measure the plant parts parameters, the experimental plants in pots were harvested after two month and the length of their parts, roots, stems and leaves was measured through scale. For fresh biomass the plants were separated into roots, stem and leaves) and weighed through physical balance. Each part was packed in envelope and labelled. The samples were kept in oven for dryness at $80^{\circ} \mathrm{C}$ for $48 \mathrm{hrs}$. Through mortar and pestle the dried samples were crushed into powdered and packed in small polythene bags.
Table I Application of $\mathrm{Cd}$ and $\mathrm{NaCl}$ during experiments. $\mathrm{C}$ for control is compared with all treatments to find out the effect of $\mathrm{Cd}$ alone and in combinations with salt $(\mathrm{NaCl})$ on plant growth. While $\mathrm{Cl}, \mathrm{C} 2$ and $\mathrm{C} 3$ are compared with all other treatments for $\mathrm{NaCl}$ effect on $\mathrm{Cd}$ phyto accumulation

\begin{tabular}{|c|c|c|c|}
\hline Treatments & Denoted & Treatments & Denoted \\
\hline $\begin{array}{l}\text { Growth media Soil } \\
\text { only }\end{array}$ & C & $100 p p m \mathrm{Cd}+1000 \mathrm{ppm} \mathrm{NaCl}$ & $\mathrm{T} 4$ \\
\hline 50ppm Cd & $\mathrm{Cl}$ & $100 p p m \mathrm{Cd}+3000 \mathrm{ppm} \mathrm{NaCl}$ & T5 \\
\hline 100ppm Cd & $\mathrm{C} 2$ & 100ppm Cd+6000ppm NaCl & T6 \\
\hline 150ppm Cd & C3 & 150ppm Cd+1000ppm NaCl & $\mathrm{T7}$ \\
\hline $\begin{array}{l}50 p p m \mathrm{Cd} \\
+1000 \mathrm{ppm} \mathrm{NaCl}\end{array}$ & TI & 150ppm Cd+3000ppm NaCl & T8 \\
\hline \multicolumn{2}{|c|}{$\begin{array}{l}\text { 50ppm Cd+3000ppm } \mathrm{T2} \\
\mathrm{NaCl}\end{array}$} & 150ppm Cd+6000ppm NaCl & T9 \\
\hline \multicolumn{3}{|c|}{$\begin{array}{l}\text { 50ppm Cd+6000ppm } \\
\mathrm{NaCl}\end{array}$} & \\
\hline
\end{tabular}

\section{Analysis of $\mathrm{Cd}$ in plant tissues after complete degradation in Acid}

$0.25 \mathrm{~g}$ from dried samples were taken in conical flask and dissolved in strong acids (Nitric acid and Sulfuric acid in ratio of 5:1) followed the method of 20, Allen (1974) with minor alteration. The flasks were kept on hot plate for 15 minutes at $300^{\circ} \mathrm{C}$ until the white fumes were come out. The acid dissolved solution was cooled, filtered into plastic bottles and for reaching volume up to $50 \mathrm{ml}$, distal water was added. 5 to $10 \mathrm{ml}$ was taken from each bottle and examined by atomic absorption spectrophotometer for $\mathrm{Cd}$ concentration in central resource lab, Peshawar.

\section{Statistical analysis}

SPSS-16 and MS-excel (2010) and graph pad prism to analyse the data for actual value of $\mathrm{Cd}$. The data was subjected to ANOVA and the mean values were compared by using Tukey's Honestly Significant Difference (HSD) test, at $\mathrm{P}<0.05$.

\section{Results}

\section{Effect of Cd on Growth, Biomass and water content of Plants}

The effect of different treatments on plants growth is shown in Figure 1. The root, stem and leaf length of Hemerocallis and Dodonaea plants are given in Tables 2, Table 3 respectively. All the plants showed significant decrease in growth, biomass and total water content under different $\mathrm{Cd}$ concentrations (50, 100 and 150ppm). This decrease was highly significant at the highest concentration of Cd (150ppm) when the control without $\mathrm{Cd}(\mathrm{C})$ was compared with $\mathrm{Cd}$ treated plants $(\mathrm{C} 1, \mathrm{C} 2$ and $\mathrm{C} 3)$ as shown in Tables 2, Table 3 respectively for Hemerocallis and Dodonaea plants. At lower concentrations of $\mathrm{Cd}$ was not statistically significant as compared to control C (Table 2). Similarly, the lowest concentration of $\mathrm{Cd}(50 \mathrm{ppm})$ shows nonsignificant decrease in all the above growth parameters (except the stem length) of Dodonaea plant as compared to the control $\mathrm{C} \pm$ (Table 3 ). The results showed a gradual decline in growth parameters in all the plants with increasing $\mathrm{Cd}$ concentration.

\section{Combine Effect of $\mathrm{Cd}$ and Salt $(\mathrm{NaCl})$ on Plant Growth and biomass}

The higher concentrations (3000 and 6000ppm) of $\mathrm{NaCl}$ salt in 
combination with $\mathrm{Cd}$ significantly decreased the growth, biomass and total water content of both Hemerocallis (Table 2 ) and Dodonaea (Table 3) plants when $\mathrm{C} 1$ (50ppm Cd in Soil) was compared with $\mathrm{T} 2$ (50ppm $\mathrm{Cd}+3000 \mathrm{ppm} \mathrm{NaCl}$ in Soil) and $\mathrm{T} 3(50 \mathrm{ppm} \mathrm{Cd}+6000 \mathrm{ppm} \mathrm{NaCl}$ in Soil). Similarly, when C2 (100ppm Cd in Soil) was compared with T5 (100ppm $\mathrm{Cd}+3000 \mathrm{ppm} \mathrm{NaCl}$ in Soil) and T6 (100ppm Cd+6000ppm $\mathrm{NaCl}$ in Soil), and C3 (150ppm Cd in Soil) when compared with T8 (150ppm Cd+3000ppm NaCl in Soil) and T9 (150ppm Cd+6000ppm $\mathrm{NaCl}$ in Soil) given in Tables $2 \& 3$. The lower concentration of $\mathrm{NaCl}(1000 \mathrm{ppm} \mathrm{NaCl}$ in Soil) in combination with $\mathrm{Cd}$ (T1, T4 and T7) showed no significant difference in all the growth parameters when compared $\mathrm{C} 1, \mathrm{C} 2$ and $\mathrm{C} 3$ respectively. The highest significant decrease in all the above growth parameters for Hemerocallis plant was recorded for the treatment T9 $(150 \mathrm{ppm} \mathrm{Cd}+6000 \mathrm{ppm} \mathrm{NaCl})$ as compared to control C. Dodonaea plant showed decrease in plant growth (root and shoot length) and biomass (fresh and dry) with increasing salts $(\mathrm{NaCl})$ concentration. This decrease was significant only at higher salt concentrations (3000 and 6000ppm) when T2, T3 was compared with $\mathrm{C} 1$ and $\mathrm{T} 5$, $\mathrm{T} 6$ was compared with $\mathrm{C} 2$, and $\mathrm{T} 8$, T9 was compared with C3 (Table 3). The highest significant decrease in all the growth parameters was recorded in the treatment $\mathrm{T} 9$ as compared to control C.

Table 2 Effect on Hemerocallis plant. C (Soil without Cd and NaCl addition), Cl, C2, C3 (50, 100, I50ppm Cd in Soil), TI, T2, T3 (1000, 3000, 6000ppm $\mathrm{NaCl}+50 \mathrm{ppm} \mathrm{Cd}$ with each $\mathrm{NaCl}$ concentration),T4,T5,T6 (1000, 3000,6000ppm NaCl+100ppm Cd),T7,T8,T9 (I000, 3000, 6000ppm NaCl+ I50ppm Cd). \pm SD denote Standard deviation and different letters show the significant difference among different treatments for a specific parameter

\begin{tabular}{|c|c|c|c|c|c|c|c|c|c|c|c|c|}
\hline \multirow{2}{*}{ Treatments } & \multicolumn{3}{|c|}{ Length $\mathrm{cm}$} & \multicolumn{3}{|c|}{ Fresh Biomass g } & \multicolumn{3}{|c|}{ Dry Biomass g } & \multicolumn{3}{|c|}{ Total Water Contents g } \\
\hline & Root & Stem & Leaves & Root & Stem & Leaves & Root & Stem & Leaves & Root & Stem & Leaves \\
\hline $\mathrm{C}$ & $15 \pm 1^{a}$ & $\begin{array}{c}6.25 \pm \\
0.25^{\mathrm{a}}\end{array}$ & $\begin{array}{c}26.3 \pm \\
1.75^{\mathrm{a}}\end{array}$ & $\begin{array}{c}10.56 \pm \\
0.32^{\mathrm{a}}\end{array}$ & $\begin{array}{c}4.448 \pm \\
0.61^{\mathrm{a}}\end{array}$ & $\begin{array}{l}18.5^{ \pm} \\
0.57^{\mathrm{a}}\end{array}$ & $\begin{array}{c}4.226 \pm \\
0.13^{\mathrm{a}}\end{array}$ & $\begin{array}{c}1.779 \pm \\
0.243^{\mathrm{a}}\end{array}$ & $\begin{array}{c}7.395 \pm \\
0.227^{\mathrm{a}}\end{array}$ & $\begin{array}{l}6.3 \pm \\
0.19^{\mathrm{a}}\end{array}$ & $\begin{array}{c}2.669 \pm \\
0.365^{\mathrm{a}}\end{array}$ & $\begin{array}{c}11.1 \pm \\
0.34^{\mathrm{a}}\end{array}$ \\
\hline $\mathrm{C} 1$ & $12 \pm 1^{\mathrm{b}}$ & $\begin{array}{l}4.50 \pm \\
0.50^{\mathrm{b}}\end{array}$ & $\begin{array}{c}21.00 \pm \\
1.75^{\mathrm{b}}\end{array}$ & $\begin{array}{c}8.65 \pm \\
1.16^{\mathrm{b}}\end{array}$ & $\begin{array}{c}3.212 \pm \\
0.19^{\mathrm{b}}\end{array}$ & $\begin{array}{l}15.1 \pm \\
2.03^{b}\end{array}$ & $\begin{array}{l}3.46^{ \pm} \\
0.46^{b}\end{array}$ & $\begin{array}{c}1.285 \pm \\
0.07^{\mathrm{b}}\end{array}$ & $\begin{array}{c}6.055 \pm \\
0.813^{b}\end{array}$ & $\begin{array}{c}5.2 \pm \\
0.7^{\mathrm{b}}\end{array}$ & $\begin{array}{c}1.927 \pm \\
0.116^{\mathrm{b}}\end{array}$ & $\begin{array}{c}9.08 \pm \\
1.22^{\mathrm{b}}\end{array}$ \\
\hline $\mathrm{C} 2$ & $\begin{array}{c}11.75 \pm \\
0.75^{\mathrm{b}}\end{array}$ & $\begin{array}{l}4.00 \pm \\
0.05^{\text {bc }}\end{array}$ & $\begin{array}{l}20.6 \pm \\
1.31^{\mathrm{b}}\end{array}$ & $\begin{array}{l}7.435 \pm \\
0.59 \text { bcd }\end{array}$ & $\begin{array}{l}2.554 \pm \\
0.37^{\text {bcd }}\end{array}$ & $\begin{array}{c}13 \pm \\
1.04 \mathrm{bcd}\end{array}$ & $\begin{array}{c}2.974 \pm \\
0.238^{\text {bcd }}\end{array}$ & $\begin{array}{l}1.022 \pm \\
0.14^{\text {bcd }}\end{array}$ & $\begin{array}{l}5.204 \pm \\
0.416^{\text {bcd }}\end{array}$ & $\begin{array}{c}4.5 \pm \\
0.36^{\text {bcd }}\end{array}$ & $\begin{array}{l}1.533 \pm \\
0.219^{\text {bcd }}\end{array}$ & $\begin{array}{l}7.81 \pm \\
0.62^{\text {bcd }}\end{array}$ \\
\hline $\mathrm{C} 3$ & $\begin{array}{c}11.25 \pm \\
1.25^{\mathrm{bc}}\end{array}$ & $\begin{array}{c}3.5 \pm \\
0.5 \\
\text { bcd }\end{array}$ & $\begin{array}{l}19.7 \pm \\
2.19^{\mathrm{bc}}\end{array}$ & $\begin{array}{l}6.373 \pm \\
0.18^{\text {cde }}\end{array}$ & $\begin{array}{l}1.974 \pm \\
0.01^{\mathrm{def}}\end{array}$ & $\begin{array}{l}11.2 \pm \\
0.32^{\text {cde }}\end{array}$ & $\begin{array}{l}2.549 \pm \\
0.074 \text { cde }\end{array}$ & $\begin{array}{c}0.79 \pm \\
0.003^{\text {def }}\end{array}$ & $\begin{array}{l}4.461 \pm \\
0.129^{\text {cde }}\end{array}$ & $\begin{array}{c}3.8 \pm \\
0.11^{\text {cde }}\end{array}$ & $\begin{array}{l}1.184 \pm \\
0.004^{\text {def }}\end{array}$ & $\begin{array}{l}6.69 \pm \\
0.19^{\text {cde }}\end{array}$ \\
\hline $\mathrm{T} 1$ & $\begin{array}{c}11.5 \pm \\
1^{\mathrm{b}}\end{array}$ & $\begin{array}{l}4.5^{ \pm} \\
0.5^{b}\end{array}$ & $\begin{array}{c}20.1 \pm \\
1.75^{\mathrm{b}}\end{array}$ & $\begin{array}{c}7.802 \pm \\
0.18 \mathrm{bc}\end{array}$ & $\begin{array}{c}3.045 \pm \\
0 \mathrm{bc}\end{array}$ & $\begin{array}{l}13.7 \pm \\
0.32 \mathrm{bc}\end{array}$ & $\begin{array}{l}3.121 \pm \\
0.073 \mathrm{bc}\end{array}$ & $\begin{array}{l}1.218 \pm \\
0.001 \mathrm{bc}\end{array}$ & $\begin{array}{l}5.461 \pm \\
0.128 \mathrm{bc}\end{array}$ & $\begin{array}{c}4.7 \pm \\
0.11^{\mathrm{bc}}\end{array}$ & $\begin{array}{l}1.827 \pm \\
0.002 \mathrm{bc}\end{array}$ & $\begin{array}{l}8.19 \pm \\
0.19 \mathrm{bc}\end{array}$ \\
\hline $\mathrm{T} 2$ & $\begin{array}{l}10.5 \pm \\
0.5^{\text {bcd }}\end{array}$ & $\begin{array}{l}3.75 \pm \\
0.25^{\mathrm{bc}}\end{array}$ & $\begin{array}{l}18.4 \pm \\
0.88^{\text {bcd }}\end{array}$ & $\begin{array}{c}6.116 \pm \\
0.34^{\mathrm{de}}\end{array}$ & $\begin{array}{l}2.18 \pm \\
0.08^{\text {cde }}\end{array}$ & $\begin{array}{l}10.7 \pm \\
0.59^{\mathrm{de}}\end{array}$ & $\begin{array}{l}2.447 \pm \\
0.134^{\text {de }}\end{array}$ & $\begin{array}{l}0.872 \pm \\
0.031^{\text {cde }}\end{array}$ & $\begin{array}{l}4.281 \pm \\
0.235^{\mathrm{de}}\end{array}$ & $\begin{array}{l}3.7 \pm \\
0.2 \text { de }\end{array}$ & $\begin{array}{l}1.308 \pm \\
0.047^{\text {cde }}\end{array}$ & $\begin{array}{l}6.42 \pm \\
0.35^{\text {de }}\end{array}$ \\
\hline $\mathrm{T} 3$ & $\begin{array}{r}8.25 \pm \\
0.75^{\mathrm{d}}\end{array}$ & $\begin{array}{l}3.25 \pm \\
0.25^{\text {cd }}\end{array}$ & $\begin{array}{l}14.4 \pm \\
1.31^{\mathrm{d}}\end{array}$ & $\begin{array}{c}5.71 \pm \\
0.2^{\mathrm{e}}\end{array}$ & $\begin{array}{l}2.297 \pm \\
0.46^{\text {bcd }}\end{array}$ & $\begin{array}{c}9.99 \pm \\
0.34^{\mathrm{e}}\end{array}$ & $\begin{array}{c}2.284 \pm \\
0.079^{\mathrm{e}}\end{array}$ & $\begin{array}{l}0.919 \pm \\
0.184^{\text {bcd }}\end{array}$ & $\begin{array}{c}3.997 \pm \\
0.138^{\mathrm{e}}\end{array}$ & $\begin{array}{l}3.4 \pm \\
0.12^{\mathrm{e}}\end{array}$ & $\begin{array}{l}1.378 \pm \\
0.276^{\text {bcd }}\end{array}$ & $\begin{array}{c}6 \pm \\
0.21^{\mathrm{e}}\end{array}$ \\
\hline $\mathrm{T} 4$ & $\begin{array}{c}11.75 \pm \\
1.25^{\mathrm{b}}\end{array}$ & $\begin{array}{l}3.6 \pm \\
0.4 b^{\text {cd }}\end{array}$ & $\begin{array}{c}20.6 \pm \\
2.19^{b}\end{array}$ & $\begin{array}{c}6.213 \pm \\
0.17^{\text {cde }}\end{array}$ & $\begin{array}{c}1.914 \pm \\
0.52^{\mathrm{def}}\end{array}$ & $\begin{array}{l}10.9 \pm \\
0.31^{\text {de }}\end{array}$ & $\begin{array}{c}2.485 \pm \\
0.07^{\text {cde }}\end{array}$ & $\begin{array}{l}0.765 \pm \\
0.208^{\text {def }}\end{array}$ & $\begin{array}{l}4.349 \pm \\
0.122^{\text {cde }}\end{array}$ & $\begin{array}{l}3.7 \pm \\
0.1^{\text {cde }}\end{array}$ & $\begin{array}{l}1.148 \pm \\
0.312^{\text {def }}\end{array}$ & $\begin{array}{l}6.52 \pm \\
0.18^{\text {cde }}\end{array}$ \\
\hline $\mathrm{T} 5$ & $\begin{array}{c}9.5 \pm \\
0.5 \text { bcd }\end{array}$ & $\begin{array}{l}3.2 \pm \\
0.2^{\mathrm{cd}}\end{array}$ & $\begin{array}{c}16.6 \pm \\
0.88^{\text {bcd }}\end{array}$ & $\begin{array}{c}4.049 \pm \\
0.81^{\mathrm{f}}\end{array}$ & $\begin{array}{c}1.366 \pm \\
0.28^{\mathrm{efg}}\end{array}$ & $\begin{array}{c}7.09 \pm \\
1.41^{\mathrm{f}}\end{array}$ & $\begin{array}{l}1.62 \pm \\
0.322^{\mathrm{f}}\end{array}$ & $\begin{array}{l}0.546 \pm \\
0.114^{\mathrm{efg}}\end{array}$ & $\begin{array}{c}2.834 \pm \\
0.564^{\mathrm{f}}\end{array}$ & $\begin{array}{l}2.4 \pm \\
0.48^{f}\end{array}$ & $\begin{array}{l}0.819 \pm \\
0.171^{\text {efg }}\end{array}$ & $\begin{array}{c}4.25 \pm \\
0.85^{f}\end{array}$ \\
\hline $\mathrm{T} 6$ & $\begin{array}{l}8.75 \pm \\
1.25^{\text {cd }}\end{array}$ & $\begin{array}{l}2.5 \pm \\
0.5^{\mathrm{de}}\end{array}$ & $\begin{array}{l}15.3 \pm \\
2.19^{\text {cd }}\end{array}$ & $\begin{array}{c}3.79 \pm \\
0.14^{\mathrm{fg}}\end{array}$ & $\begin{array}{c}1.076 \pm \\
0.1^{1 \mathrm{~g}}\end{array}$ & $\begin{array}{l}6.63 \pm \\
0.25^{\mathrm{fg}}\end{array}$ & $\begin{array}{l}1.516 \pm \\
0.058^{\mathrm{fg}}\end{array}$ & $\begin{array}{l}0.431 \pm \\
0.042^{\mathrm{fg}}\end{array}$ & $\begin{array}{l}2.653 \pm \\
0.101^{\mathrm{fg}}\end{array}$ & $\begin{array}{l}2.3 \pm \\
0.09^{f g}\end{array}$ & $\begin{array}{l}0.646 \pm \\
0.062^{ \pm g}\end{array}$ & $\begin{array}{c}3.98 \pm \\
0.15^{\mathrm{fg}}\end{array}$ \\
\hline $\mathrm{T} 7$ & $12 \pm 1^{\mathrm{b}}$ & $\begin{array}{l}3.5 \pm \\
0.5^{\text {bcd }}\end{array}$ & $\begin{array}{c}21 \pm \\
1.75^{\mathrm{b}}\end{array}$ & $\begin{array}{c}6.085 \pm \\
0.62^{\mathrm{de}}\end{array}$ & $\begin{array}{l}1.767 \pm \\
0.22^{\text {defg }}\end{array}$ & $\begin{array}{l}10.6 \pm \\
1.09^{\text {de }}\end{array}$ & $\begin{array}{c}2.434 \pm \\
0.25^{\mathrm{de}}\end{array}$ & $\begin{array}{c}0.707 \pm \\
0.087^{\text {defg }}\end{array}$ & $\begin{array}{l}4.259 \pm \\
0.437^{\text {de }}\end{array}$ & $\begin{array}{c}3.7 \pm \\
0.37^{\mathrm{de}}\end{array}$ & $\begin{array}{c}1.06 \pm \\
0.131^{\text {defg }}\end{array}$ & $\begin{array}{l}6.39 \pm \\
0.66^{\text {de }}\end{array}$ \\
\hline $\mathrm{T} 8$ & $8.5 \pm 0^{\mathrm{d}}$ & $2 \pm 0^{\mathrm{e}}$ & $\begin{array}{c}14.9 \pm \\
0^{\mathrm{d}}\end{array}$ & $\begin{array}{c}3.852 \pm \\
0.84^{\mathrm{fg}}\end{array}$ & $\begin{array}{c}0.906 \pm \\
0.2^{\mathrm{g}}\end{array}$ & $\begin{array}{l}6.74 \pm \\
1.47^{\mathrm{fg}}\end{array}$ & $\begin{array}{l}1.541 \pm \\
0.335^{\mathrm{fg}}\end{array}$ & $\begin{array}{c}0.363 \pm \\
0.079^{g}\end{array}$ & $\begin{array}{c}2.697 \pm \\
0.586^{\mathrm{fg}}\end{array}$ & $\begin{array}{l}2.3 \pm \\
0.5^{\mathrm{fg}}\end{array}$ & $\begin{array}{c}0.544 \pm \\
0.118^{g}\end{array}$ & $\begin{array}{l}4.04 \pm \\
0.88^{f g}\end{array}$ \\
\hline T9 & $\begin{array}{c}4.25 \pm \\
0.75^{\mathrm{e}}\end{array}$ & $\begin{array}{l}1.45 \pm \\
0.05^{\mathrm{e}}\end{array}$ & $\begin{array}{c}7.44 \pm \\
1.31^{\mathrm{e}}\end{array}$ & $\begin{array}{c}2.414 \pm \\
0.1^{\mathrm{g}}\end{array}$ & $\begin{array}{c}0.863 \pm \\
0.22^{g}\end{array}$ & $\begin{array}{c}4.22 \pm \\
0.18^{\mathrm{g}}\end{array}$ & $\begin{array}{c}0.965 \pm \\
0.041^{\mathrm{g}}\end{array}$ & $\begin{array}{c}0.345 \pm \\
0.086^{\mathrm{g}}\end{array}$ & $\begin{array}{c}1.689 \pm \\
0.072^{\mathrm{g}}\end{array}$ & $\begin{array}{l}1.4 \pm \\
0.06^{g}\end{array}$ & $\begin{array}{c}0.518 \pm \\
0.13^{\mathrm{g}}\end{array}$ & $\begin{array}{r}2.53 \pm \\
0.11^{\mathrm{g}}\end{array}$ \\
\hline
\end{tabular}


Table 3 Dodonaea plant growth. C (Soil without Cd and NaCl addition), CI, C2, C3 (50, I00, I50ppm Cd in Soil), TI,T2,T3 (I000, 3000, 6000ppm NaCl+50ppm Cd with each $\mathrm{NaCl}$ concentration), T4, T5, T6 (I000, 3000, 6000ppm NaCl+ I00ppm Cd), T7, T8, T9 (I000, 3000, 6000ppm NaCl+I50ppm Cd). \pm SD denote Standard deviation and different letters show the significant difference among different treatments for a specific parameter.

\begin{tabular}{|c|c|c|c|c|c|c|c|c|c|c|c|c|}
\hline \multirow{2}{*}{ Treatments } & \multicolumn{3}{|c|}{ Length $(\mathrm{cm}) \pm \mathrm{SD}$} & \multicolumn{3}{|c|}{ Fresh Biomass (g) \pm SD } & \multicolumn{3}{|c|}{ Dry Biomass (g) \pm SD } & \multicolumn{3}{|c|}{ Total Water Contents $(g)^{ \pm}$SD } \\
\hline & Root & Stem & Leaves & Root & Stem & Leaves & Root & Stem & Leaves & Root & Stem & Leaves \\
\hline $\mathrm{C}$ & $\begin{array}{c}25.00 \pm \\
1.00^{\mathrm{a}}\end{array}$ & $\begin{array}{c}35.00 \pm \\
1.00^{\mathrm{a}}\end{array}$ & $\begin{array}{c}9.00 \pm \\
1.00^{\mathrm{a}}\end{array}$ & $\begin{array}{c}1.98 \pm \\
0.07^{\mathrm{a}}\end{array}$ & $\begin{array}{c}2.79 \pm \\
0.29^{\mathrm{a}}\end{array}$ & $\begin{array}{c}0.72 \pm \\
0.13^{\mathrm{a}}\end{array}$ & $\begin{array}{c}0.79 \pm \\
0.03^{\mathrm{a}}\end{array}$ & $\begin{array}{c}1.12 \pm \\
0.12^{\mathrm{a}}\end{array}$ & $\begin{array}{c}0.28 \pm \\
0.05^{\mathrm{a}}\end{array}$ & $\begin{array}{c}1.19 \pm \\
0.04^{\mathrm{a}}\end{array}$ & $\begin{array}{c}1.67 \pm \\
0.17^{\mathrm{a}}\end{array}$ & $\begin{array}{c}0.43 \pm \\
0.08^{\mathrm{a}}\end{array}$ \\
\hline $\mathrm{C} 1$ & $\begin{array}{c}22.50 \pm \\
0.50^{\mathrm{ab}}\end{array}$ & $\begin{array}{c}29.50 \pm \\
0.50^{b}\end{array}$ & $\begin{array}{l}7.50 \pm \\
1.50^{\mathrm{ab}}\end{array}$ & $\begin{array}{l}1.79 \pm \\
0.04^{\mathrm{ab}}\end{array}$ & $\begin{array}{l}2.35 \pm \\
0.06^{\mathrm{ab}}\end{array}$ & $\begin{array}{l}0.60 \pm \\
0.14^{\mathrm{ab}}\end{array}$ & $\begin{array}{l}0.72 \pm \\
0.02^{\mathrm{ab}}\end{array}$ & $\begin{array}{c}0.94 \pm \\
0.03^{\mathrm{ab}}\end{array}$ & $\begin{array}{c}0.24 \pm \\
0.05^{\mathrm{ab}}\end{array}$ & $\begin{array}{l}1.07 \pm \\
0.02^{\mathrm{ab}}\end{array}$ & $\begin{array}{l}1.41 \pm \\
0.03^{\mathrm{ab}}\end{array}$ & $\begin{array}{c}0.36 \pm \\
0.08^{\mathrm{ab}}\end{array}$ \\
\hline $\mathrm{C} 2$ & $\begin{array}{c}21.50 \pm \\
2.50^{\mathrm{abc}}\end{array}$ & $\begin{array}{c}22.50 \pm \\
2.50^{c}\end{array}$ & $\begin{array}{c}5.00 \pm \\
0.01^{\text {bcde }}\end{array}$ & $\begin{array}{l}1.54 \pm \\
0.24^{\mathrm{bc}}\end{array}$ & $\begin{array}{l}1.61 \pm \\
0.24^{\text {cdef }}\end{array}$ & $\begin{array}{c}0.35 \pm \\
0.01^{\text {cdef }}\end{array}$ & $\begin{array}{c}0.61 \pm \\
0.1^{\mathrm{bc}}\end{array}$ & $\begin{array}{l}0.64 \pm \\
0.10^{\text {cde }}\end{array}$ & $\begin{array}{l}0.14^{ \pm} \\
0.01^{\text {cdef }}\end{array}$ & $\begin{array}{l}0.92 \pm \\
0.14^{\mathrm{bc}}\end{array}$ & $\begin{array}{l}0.96 \pm \\
0.14^{\text {cde }}\end{array}$ & $\begin{array}{l}0.21 \pm \\
0.01^{\text {cdef }}\end{array}$ \\
\hline $\mathrm{C} 3$ & $\begin{array}{c}19.50 \pm \\
0.50^{\mathrm{bc}}\end{array}$ & $\begin{array}{c}21.00 \pm \\
1.00^{c}\end{array}$ & $\begin{array}{l}4.50 \pm \\
0.50^{\text {cde }}\end{array}$ & $\begin{array}{l}1.52 \pm \\
0.22 \mathrm{bc}\end{array}$ & $\begin{array}{l}1.63 \pm \\
0.27^{\text {cdef }}\end{array}$ & $\begin{array}{l}0.34 \pm \\
0.01^{\text {def }}\end{array}$ & $\begin{array}{l}0.61 \pm \\
0.09^{\mathrm{bc}}\end{array}$ & $\begin{array}{l}0.66 \pm \\
0.11^{\text {cd }}\end{array}$ & $\begin{array}{l}0.13 \pm \\
0.01^{\text {def }}\end{array}$ & $\begin{array}{l}0.91 \pm \\
0.13^{\mathrm{bc}}\end{array}$ & $\begin{array}{l}0.98 \pm \\
0.16^{\mathrm{cd}}\end{array}$ & $\begin{array}{c}0.20 \\
\pm 0.01^{\text {def }}\end{array}$ \\
\hline $\mathrm{T} 1$ & $\begin{array}{c}21.00 \pm \\
1.00^{\mathrm{abc}}\end{array}$ & $\begin{array}{c}29.00 \pm \\
1.00^{\mathrm{b}}\end{array}$ & $\begin{array}{l}7.50 \pm \\
1.50^{\mathrm{ab}}\end{array}$ & $\begin{array}{l}1.65 \pm \\
0.20^{\mathrm{ab}}\end{array}$ & $\begin{array}{l}2.28 \pm \\
0.31^{\mathrm{ab}}\end{array}$ & $\begin{array}{l}0.57 \pm \\
0.02^{\mathrm{abc}}\end{array}$ & $\begin{array}{l}0.66 \pm \\
0.08^{\mathrm{ab}}\end{array}$ & $\begin{array}{l}0.91 \pm \\
0.13^{\mathrm{ab}}\end{array}$ & $\begin{array}{l}0.23 \pm \\
0.01^{\mathrm{abc}}\end{array}$ & $\begin{array}{l}0.98 \pm \\
0.12^{\text {ab }}\end{array}$ & $\begin{array}{l}1.36 \pm \\
0.18^{\mathrm{ab}}\end{array}$ & $\begin{array}{c}0.34 \pm \\
0.01^{\mathrm{abc}}\end{array}$ \\
\hline $\mathrm{T} 2$ & $\begin{array}{c}14.50 \pm \\
0.50^{\mathrm{de}}\end{array}$ & $\begin{array}{c}15.00 \pm \\
1.00^{\mathrm{d}}\end{array}$ & $\begin{array}{l}4.00 \pm \\
0.01^{\mathrm{de}}\end{array}$ & $\begin{array}{l}1.43 \pm \\
0.14 \text { bc }\end{array}$ & $\begin{array}{l}1.47 \pm \\
0.10^{\text {cdef }}\end{array}$ & $\begin{array}{c}0.39 \pm \\
0.05^{\text {bcdef }}\end{array}$ & $\begin{array}{l}0.57 \pm \\
0.06^{\mathrm{bc}}\end{array}$ & $\begin{array}{l}0.59 \pm \\
0.04^{\text {cdef }}\end{array}$ & $\begin{array}{c}0.16 \pm \\
0.02^{\text {bcdef }}\end{array}$ & $\begin{array}{l}0.85 \pm \\
0.08^{\mathrm{bc}}\end{array}$ & $\begin{array}{c}0.88 \pm \\
0.06^{\text {cdef }}\end{array}$ & $\begin{array}{c}0.23 \pm \\
0.03^{\text {bcdef }}\end{array}$ \\
\hline T3 & $\begin{array}{c}12.50 \pm \\
2.50^{\mathrm{ef}}\end{array}$ & $\begin{array}{c}13.00 \pm \\
3.00^{\mathrm{d}}\end{array}$ & $\begin{array}{l}3.85 \pm \\
0.35^{\text {de }}\end{array}$ & $\begin{array}{c}1.17 \pm \\
0.14^{\mathrm{c}}\end{array}$ & $\begin{array}{l}1.21 \pm \\
0.18^{\text {def }}\end{array}$ & $\begin{array}{c}0.36 \pm \\
0.06^{\text {cdef }}\end{array}$ & $\begin{array}{l}0.47^{ \pm} \\
0.05^{c}\end{array}$ & $\begin{array}{l}0.48 \pm \\
0.07^{\text {def }}\end{array}$ & $\begin{array}{l}0.15 \pm \\
0.02^{\text {cdef }}\end{array}$ & $\begin{array}{c}0.70 \pm \\
0.08^{\mathrm{c}}\end{array}$ & $\begin{array}{l}0.72 \pm \\
0.10^{\text {def }}\end{array}$ & $\begin{array}{l}0.22 \pm \\
0.03^{\text {cdef }}\end{array}$ \\
\hline $\mathrm{T} 4$ & $\begin{array}{c}18.00 \pm \\
2.00^{\mathrm{cd}}\end{array}$ & $\begin{array}{c}22.52 \pm \\
0.50^{c}\end{array}$ & $\begin{array}{c}7.00 \pm \\
2.00^{\mathrm{abc}}\end{array}$ & $\begin{array}{l}1.46 \pm \\
0.11^{\mathrm{bc}}\end{array}$ & $\begin{array}{l}1.83 \pm \\
0.10^{\mathrm{bc}}\end{array}$ & $\begin{array}{c}0.56 \pm \\
0.14^{\text {abcd }}\end{array}$ & $\begin{array}{l}0.58 \pm \\
0.04^{\mathrm{bc}}\end{array}$ & $\begin{array}{l}0.73 \pm \\
0.04^{\mathrm{bc}}\end{array}$ & $\begin{array}{c}0.22 \pm \\
0.05^{\text {abcd }}\end{array}$ & $\begin{array}{l}0.87 \pm \\
0.06^{\mathrm{bc}}\end{array}$ & $\begin{array}{l}1.09 \pm \\
0.06^{\mathrm{bc}}\end{array}$ & $\begin{array}{c}0.33 \pm \\
0.08 \text { abcd }\end{array}$ \\
\hline $\mathrm{T} 5$ & $\begin{array}{l}9.50 \pm \\
1.50^{\mathrm{fg}}\end{array}$ & $\begin{array}{c}12.50 \pm \\
2.50^{\mathrm{d}}\end{array}$ & $\begin{array}{l}4.00 \pm \\
0.01^{\mathrm{de}}\end{array}$ & $\begin{array}{c}0.73 \pm \\
0.01^{\mathrm{d}}\end{array}$ & $\begin{array}{c}0.90 \pm \\
0.06^{\mathrm{fg}}\end{array}$ & $\begin{array}{l}0.31 \pm \\
0.04^{\mathrm{ef}}\end{array}$ & $\begin{array}{c}0.29 \pm \\
0.01^{\mathrm{d}}\end{array}$ & $\begin{array}{c}0.40 \pm \\
0.02^{\mathrm{fg}}\end{array}$ & $\begin{array}{c}0.13 \pm \\
0.01^{\text {ef }}\end{array}$ & $\begin{array}{c}0.43 \pm \\
0.02^{\mathrm{d}}\end{array}$ & $\begin{array}{c}0.54 \pm \\
0.02^{\mathrm{fg}}\end{array}$ & $\begin{array}{c}0.18 \pm \\
0.03^{\text {ef }}\end{array}$ \\
\hline T6 & $\begin{array}{c}8.50 \pm \\
0.50^{\mathrm{fg}}\end{array}$ & $\begin{array}{c}11.50 \pm \\
0.50^{\text {de }}\end{array}$ & $\begin{array}{l}4.03 \pm \\
0.07^{\text {de }}\end{array}$ & $\begin{array}{c}0.71 \pm \\
0.01^{\mathrm{d}}\end{array}$ & $\begin{array}{c}0.95 \\
\pm 0.01^{\mathrm{fg}}\end{array}$ & $\begin{array}{c}0.33 \pm \\
0.01^{\text {ef }}\end{array}$ & $\begin{array}{c}0.28 \pm \\
0.01^{\mathrm{d}}\end{array}$ & $\begin{array}{c}0.38 \pm \\
0.01^{\mathrm{fg}}\end{array}$ & $\begin{array}{c}0.13 \pm \\
0.01^{\text {ef }}\end{array}$ & $\begin{array}{c}0.42 \pm \\
0.07^{\mathrm{d}}\end{array}$ & $\begin{array}{l}0.57 \pm \\
0.01^{\mathrm{fg}}\end{array}$ & $\underset{\text { ef }}{0.2 \pm 0.01}$ \\
\hline $\mathrm{T} 7$ & $\begin{array}{c}14.50 \pm \\
1.50^{\mathrm{de}}\end{array}$ & $\begin{array}{c}22.50 \pm \\
2.50^{\mathrm{c}}\end{array}$ & $\begin{array}{c}6.50 \pm \\
0.50^{\text {abcd }}\end{array}$ & $\begin{array}{c}1.17 \pm \\
0.19^{c}\end{array}$ & $\begin{array}{l}1.81 \pm \\
0.31^{\mathrm{bc}}\end{array}$ & $\begin{array}{c}0.51 \pm \\
0.01^{\text {abcde }}\end{array}$ & $\begin{array}{c}0.47 \pm \\
0.08^{\mathrm{c}}\end{array}$ & $\begin{array}{c}0.73 \pm \\
0.13^{\mathrm{bc}}\end{array}$ & $\begin{array}{c}0.20 \pm \\
0.01^{\text {abcde }}\end{array}$ & $\begin{array}{c}0.70 \pm \\
0.11^{\mathrm{c}}\end{array}$ & $\begin{array}{l}1.08 \pm \\
0.18^{\mathrm{bc}}\end{array}$ & $\begin{array}{c}0.31 \pm \\
0.01 \text { abcde }\end{array}$ \\
\hline $\mathrm{T} 8$ & $\begin{array}{c}7.50 \pm \\
0.50^{\mathrm{g}}\end{array}$ & $\begin{array}{c}12.00 \pm \\
2.00^{\mathrm{de}}\end{array}$ & $\begin{array}{l}3.65 \pm \\
0.65^{\mathrm{ef}}\end{array}$ & $\begin{array}{c}0.65 \pm \\
0.07^{\mathrm{d}}\end{array}$ & $\begin{array}{l}1.03 \pm \\
0.13^{\text {efg }}\end{array}$ & $\begin{array}{c}0.30 \pm \\
0.06^{\mathrm{ef}}\end{array}$ & $\begin{array}{c}0.26 \pm \\
0.03^{\mathrm{d}}\end{array}$ & $\begin{array}{l}0.41 \pm \\
0.05^{\text {efg }}\end{array}$ & $\begin{array}{c}0.12 \pm \\
0.02^{\text {ef }}\end{array}$ & $\begin{array}{c}0.39 \pm \\
0.04^{\mathrm{d}}\end{array}$ & $\begin{array}{l}0.61 \pm \\
0.08^{\text {efg }}\end{array}$ & $0.04^{\mathrm{ef}}$ \\
\hline T9 & $\begin{array}{c}7.00 \pm \\
1.00^{\mathrm{g}}\end{array}$ & $\begin{array}{c}7.50 \pm \\
0.50^{\mathrm{e}}\end{array}$ & $\begin{array}{l}3.10 \pm \\
0.10^{\mathrm{ef}}\end{array}$ & $\begin{array}{l}0.58 \pm \\
0.09^{\mathrm{d}}\end{array}$ & $\begin{array}{c}0.61 \pm \\
0.03^{\mathrm{g}}\end{array}$ & $\begin{array}{c}0.25 \pm \\
0.01^{\mathrm{f}}\end{array}$ & $\begin{array}{c}0.23 \pm \\
0.04^{\mathrm{d}}\end{array}$ & $\begin{array}{c}0.25 \pm \\
0.01^{\mathrm{g}}\end{array}$ & $\begin{array}{c}0.10 \pm \\
0.01^{\mathrm{f}}\end{array}$ & $\begin{array}{c}0.34 \pm \\
0.05^{\mathrm{d}}\end{array}$ & $\begin{array}{c}0.37 \pm \\
0.02^{\mathrm{g}}\end{array}$ & $\begin{array}{c}0.15 \\
\pm 0.01^{\mathrm{f}}\end{array}$ \\
\hline
\end{tabular}

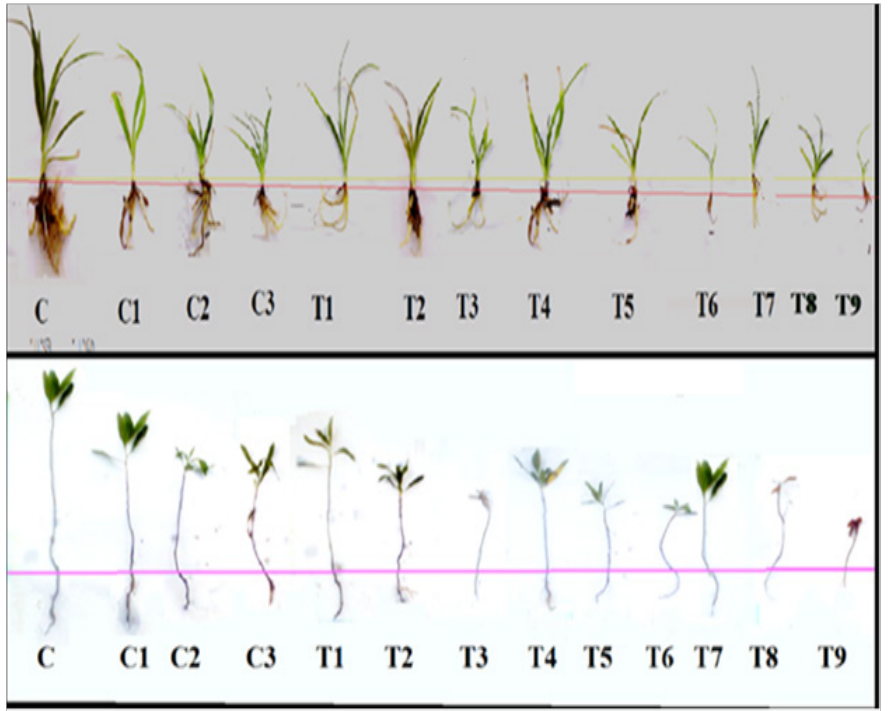

Figure I Effect of different treatments on plants growth. C (Soil without Cd and $\mathrm{NaCl}$ addition), CI, C2, C3 (50, 100, I50ppm Cd in Soil), TI, T2, T3 (I000, 3000,6000 ppm NaCl +50 ppm Cd with each $\mathrm{NaCl}$ concentration), T4, T5, T6 (I000, 3000, 6000ppm NaCl + I00ppm Cd), T7, T8, T9 (I000, 3000, 6000ppm $\mathrm{NaCl}+$ I50ppm Cd).

\section{Cadmium Concentration and Accumulation in Plants}

Hemerocallis plant showed a significant increase in tissues (Root, Stem and Leaves) $\mathrm{Cd}$ concentration with increasing $\mathrm{Cd}$ concentration $(50,100$ and 150ppm) in soil, when compared C1, C2 and C3 in Table 3 (A). Similarly, the total $\mathrm{Cd}$ accumulation in different parts of the plant also increased as the $\mathrm{Cd}$ concentration in soil was increased, but this increase was statistically not significant. Salt $(\mathrm{NaCl})$ showed positive and significant effect on $\mathrm{Cd}$ concentration and accumulation in various parts of the plant (Table 4). Increasing $\mathrm{Cd}$ and sodium salt concentration in the soil increased the Cd concentration in different parts of the plant and thus the highest significant $\mathrm{Cd}$ concentration (Root " $25.40 \pm 2.30 \mathrm{ppm}$ ", Stem “46 $\pm 2.86 \mathrm{ppm}$ " and leaf " $51 \pm 3.00 \mathrm{ppm}$ ") was recorded for the treatment T9 $(150 \mathrm{ppm} \mathrm{Cd}+6000 \mathrm{ppm} \mathrm{NaCl})$.

The highest $\mathrm{Cd}$ accumulation $(\mathrm{mg} / \mathrm{DBM})$ in root $(0.02 \pm 0.009 \mathrm{mg} /$ DBM $)$, stem $(0.016 \pm 0.003 \mathrm{mg} / \mathrm{DBM})$ and entire plant $(0.127 \pm 0.04 \mathrm{mg} /$ DBM) was observed in treatment T9, while in leaves $(0.104 \pm 0.0 \mathrm{mg} /$ DBM) it was observed in T4 (100ppm Cd, without addition of $\mathrm{NaCl}$ salt in soil). Increasing $\mathrm{Cd}$ concentration in soil increased the $\mathrm{Cd}$ accumulation percentage in stem while decreased this percentage in roots and leaves when compared C1, C2 with C3 (Table 4). The highest $\mathrm{Cd}$ percentage in roots $(25.80 \pm 1.18 \%)$ was recorded for treatment $\mathrm{T} 7$ (150ppm $\mathrm{Cd}+1000 \mathrm{ppm} \mathrm{NaCl}$ in Soil), in stem $(14.27 \pm 3.80 \%)$ for $\mathrm{C} 1$ (50ppm Cd in Soil) and in leave $(72.26 \pm 1.56 \%)$ for $\mathrm{T} 8(150 \mathrm{ppm} \mathrm{Cd}$ 
and 3000ppm $\mathrm{NaCl}$ in Soil). The treatment T6 showed the highest translocation factors 2.21 root-stem and 2.29 root- leaves) and bioaccumulation factor $(0.19)$ as shown in Table 4 .

Table 5 presents the $\mathrm{Cd}$ concentration and accumulation in Dodoneae plant. The highest $\mathrm{Cd}$ concentration in roots $(32.00 \pm$ $0.94 \mathrm{ppm}$ ) of the plant was found in C3 (150ppm Cd only) while in stem $(46.00 \pm 2.86 \mathrm{ppm})$ and leaves $(51.00 \pm 3.00 \mathrm{ppm})$ it was recorded for the treatment T9 $(150 \mathrm{ppm} \mathrm{Cd}+6000 \mathrm{ppm} \mathrm{NaCl})$. Increasing the salt $(\mathrm{NaCl})$ concentration in soil increased the $\mathrm{Cd}$ concentration in different parts of the plant (Table 5). The plant accumulated more than $60 \%$ of its $\mathrm{Cd}$ in leaves in all treatments. The highest $\mathrm{Cd}$ translocation factor (2.21 roots to stem and 2.29 root to leaves) was recorded for the treatment $\mathrm{T} 6(100 \mathrm{ppm} \mathrm{Cd}+6000 \mathrm{ppm} \mathrm{NaCl}$ in Soil). The bio concentration factor of the Hemerocallis plant was much less than one (1) for all treatments (Table 5).

The highest significant $\mathrm{Cd}$ concentration in roots $(74.8 \pm 2.86$ $\& 78.4 \pm 1.36 \mathrm{ppm})$ and stem $(62.2 \pm 1.58 \& 64.4 \pm 0.9 \mathrm{ppm})$ of Dodonaea viscosa plant was observed in C2 (100ppm Cd in Soil) and C3 (200ppm Cd in Soil) in Table 3 (C). The stem showed significantly high $\mathrm{Cd}$ concentration $(62.00 \pm 1.54 \& 63.4 \pm 1.58 \mathrm{ppm})$ in treatments $\mathrm{T} 2(50 \mathrm{ppm} \mathrm{Cd}+3000 \mathrm{ppm} \mathrm{NaCl}$ in Soil) and T3 (50ppm Cd+6000ppm
$\mathrm{NaCl}$ in Soil). While leaves possess significantly the highest concentration $(98.2 \pm 1.56 \mathrm{ppm})$ of $\mathrm{Cd}$ in treatment $\mathrm{T} 3$ as shown in Table 5. The treatment $\mathrm{T} 1(50 \mathrm{ppm} \mathrm{Cd}+1000 \mathrm{ppm} \mathrm{NaCl}$ in Soil) showed the highest root to stem translocation factor (1.22). The root to leaves translocation factor (1.69) was highest in Dodonaea viscosa plant treated with $50 \mathrm{ppm} \mathrm{Cd}+6000 \mathrm{ppm} \mathrm{NaCl}$ in Soil (T3). Also the Cadmium bio-concentration factor (1.32) was found highest in the treatment T3 (Table 3).

\section{Correlation between plant $\mathrm{Cd}$ concentration and dry biomass}

Figure 2 shows correlations between dry biomass of different parts (root, stem and leaves) of Dodoneae and Hemerocallis plant species with $\mathrm{Cd}$ concentration. Negative correlation found between the dry biomass and $\mathrm{Cd}$ concentration in the root, stem and leaves of Hemerocallis plant while the roots of Dodonaea plant possessed a week positive correlation between dry biomass and $\mathrm{Cd}$ concentration but negative correlation present in its stem and leaves. From the above results it is stated that increasing of Cd concentrations automatically decreases plant dry biomasses but certain plants showed a little tolerance to such physiological stress conditions.

Table 4 Cadmium concentration and accumulation by various parts of Hemerocallis grown in soil having different concentrations of $\mathrm{NaCl}$ and cadmium $\mathrm{Cl}, \mathrm{C2}$, C3 (50, 100, 150ppm Cd in Soil), TI, T2, T3 (1000, 3000, 6000ppm NaCl+50ppm Cd with each NaCl concentration), T4, T5, T6 (I000, 3000, 6000ppm $\mathrm{NaCl}+100 \mathrm{ppm} \mathrm{Cd}), \mathrm{T} 7, \mathrm{~T} 8, \mathrm{~T} 9(1000,3000,6000 \mathrm{ppm} \mathrm{NaCl}+150 \mathrm{ppm} \mathrm{Cd}) \pm S D$ denote Standard deviation and different letters show the significant difference among different treatments for a specific parameter. R, Roots; S, stem; L, Leaves

\begin{tabular}{|c|c|c|c|c|c|c|c|c|c|c|c|c|c|}
\hline \multirow{2}{*}{ Treatments } & \multicolumn{3}{|c|}{ Cd conc. (ppm) } & \multicolumn{3}{|c|}{ Cd (mg/DBM) } & \multirow{2}{*}{$\begin{array}{c}\text { Entire } \\
\text { Plant Cd } \\
\text { Accum } \\
\text {-ulation } \\
\text { (mg/ } \\
\text { DBM) }\end{array}$} & \multicolumn{3}{|c|}{ Cd accumulation \% } & \multicolumn{2}{|c|}{$\begin{array}{c}\text { Translocation } \\
\text { Factor }\end{array}$} & \multirow{2}{*}{$\begin{array}{c}\text { Bio- } \\
\text { concentration } \\
\text { Factor }\end{array}$} \\
\hline & Roots & Stem & Leaves & $\mathbf{R}$ & $\mathbf{S}$ & $\mathbf{L}$ & & $\mathbf{R}$ & $\mathbf{S}$ & $\mathbf{L}$ & Root- Stem & $\begin{array}{l}\text { Root- } \\
\text { Leave }\end{array}$ & \\
\hline $\mathrm{C} 1$ & $\begin{array}{c}23.00 \\
\pm \\
1.75^{\text {bcd }}\end{array}$ & $\begin{array}{c}34.80 \\
\pm \\
0.38^{\mathrm{cd}}\end{array}$ & $\begin{array}{c}32.00 \pm \\
1.00^{\text {de }}\end{array}$ & $\begin{array}{l}0.08 \pm \\
0.021^{\text {a }}\end{array}$ & $\begin{array}{c}0.044 \\
\pm \\
0.003^{\mathrm{a}}\end{array}$ & $\begin{array}{c}0.194 \\
\pm 0^{\mathrm{a}}\end{array}$ & $\begin{array}{c}0.319 \pm \\
0.06^{\mathrm{a}}\end{array}$ & $\begin{array}{c}24.9 \pm \\
2^{\mathrm{bc}}\end{array}$ & $\begin{array}{c}14.27 \\
\pm \\
3.80^{\mathrm{a}}\end{array}$ & $\begin{array}{c}60.79 \\
\pm 1.80^{\mathrm{e}}\end{array}$ & 1.52 & 1.4 & 0.59 \\
\hline $\mathrm{C} 2$ & $\begin{array}{c}26.00 \\
\pm \\
2.48^{\mathrm{ab}}\end{array}$ & $\begin{array}{c}40.00 \\
\pm \\
0.54^{\mathrm{bc}}\end{array}$ & $\begin{array}{c}43.20 \pm \\
1.00^{\mathrm{bc}}\end{array}$ & $\begin{array}{c}0.08 \pm \\
0.011^{\mathrm{ab}}\end{array}$ & $\begin{array}{c}0.041 \\
\pm \\
0.002^{\mathrm{ab}}\end{array}$ & $\begin{array}{c}0.225 \\
\pm 0^{\mathrm{a}}\end{array}$ & $\begin{array}{c}0.343 \pm \\
0.04^{\mathrm{a}}\end{array}$ & $\begin{array}{l}22.6 \pm \\
1.87^{\text {bcde }}\end{array}$ & $\begin{array}{c}11.87 \\
\pm \\
2.75^{\mathrm{abc}}\end{array}$ & $\begin{array}{c}65.57 \\
\pm \\
1.41^{\text {bcde }}\end{array}$ & 1.54 & 1.66 & 0.37 \\
\hline $\mathrm{C} 3$ & $\begin{array}{c}32.00 \\
\pm \\
0.94^{\mathrm{a}}\end{array}$ & $\begin{array}{c}43.40 \\
\pm \\
2.38^{\mathrm{ab}}\end{array}$ & $\begin{array}{c}48.00 \pm \\
2.00^{\mathrm{ab}}\end{array}$ & $\begin{array}{l}0.08 \pm \\
0.007^{\mathrm{a}}\end{array}$ & $\begin{array}{c}0.0343 \\
\pm \\
0.007^{\mathrm{b}}\end{array}$ & $\begin{array}{c}0.214 \\
\pm 0^{\mathrm{a}}\end{array}$ & $\begin{array}{c}0.33 \pm \\
0.04^{\mathrm{a}}\end{array}$ & $\begin{array}{l}24.7 \pm \\
0.27^{\mathrm{bc}}\end{array}$ & $\begin{array}{c}10.39 \\
\pm \\
0.79^{\mathrm{abc}}\end{array}$ & $\begin{array}{c}64.89 \\
\pm \\
0.53^{\text {cde }}\end{array}$ & 1.36 & 1.5 & 0.28 \\
\hline $\mathrm{T} 1$ & $\begin{array}{c}12.00 \\
\pm \\
1.60^{\mathrm{e}}\end{array}$ & $\begin{array}{c}11.40 \\
\pm \\
1.28^{\mathrm{g}}\end{array}$ & $\begin{array}{c}17.40 \\
\pm 2.00^{\mathrm{h}}\end{array}$ & $\begin{array}{l}0.04 \pm \\
0.003^{\text {cd }}\end{array}$ & $\begin{array}{c}0.0139 \\
\pm \\
0.001^{\text {cd }}\end{array}$ & $\begin{array}{c}0.095 \\
\pm 0^{\mathrm{b}}\end{array}$ & $\begin{array}{c}0.146 \pm \\
0.01 \text { bc }\end{array}$ & $\begin{array}{c}25.6 \pm \\
0.27^{\mathrm{b}}\end{array}$ & $\begin{array}{l}9.47 \pm \\
0.26^{\mathrm{abc}}\end{array}$ & $\begin{array}{c}64.87 \\
\pm \\
0.38^{\text {cde }}\end{array}$ & 0.95 & 1.45 & 0.3 \\
\hline $\mathrm{T} 2$ & $\begin{array}{c}13.00 \\
\pm \\
0.88^{\mathrm{e}}\end{array}$ & $\begin{array}{c}14.20 \\
\pm \\
1.42^{\mathrm{g}}\end{array}$ & $\begin{array}{c}24.80 \pm \\
2.00^{\mathrm{efg}}\end{array}$ & $\begin{array}{c}0.03 \pm \\
0.003^{\text {cd }}\end{array}$ & $\begin{array}{c}0.0124 \\
\pm \\
0.002^{\text {cd }}\end{array}$ & $\begin{array}{c}0.106 \\
\pm 0^{\mathrm{b}}\end{array}$ & $\begin{array}{l}0.151 \pm \\
0.02^{\mathrm{bc}}\end{array}$ & $\begin{array}{c}21 \pm \\
0.83^{\text {bcde }}\end{array}$ & $\begin{array}{l}8.30 \pm \\
0.18^{\mathrm{bc}}\end{array}$ & $\begin{array}{c}70.68 \\
\pm \\
0.78^{\mathrm{ab}}\end{array}$ & 1.11 & 1.93 & 0.4 \\
\hline T3 & $\begin{array}{c}16.80 \\
\pm \\
2.72^{\mathrm{de}}\end{array}$ & $\begin{array}{c}16.00 \\
\pm \\
1.00^{\mathrm{g}}\end{array}$ & $\begin{array}{c}30.40 \pm \\
3.00^{\text {def }}\end{array}$ & $\begin{array}{l}0.04 \pm \\
0.007^{\mathrm{cd}}\end{array}$ & $\begin{array}{c}0.0146 \\
\pm \\
0.001^{\mathrm{cd}}\end{array}$ & $\begin{array}{c}0.121 \\
\pm 0^{\mathrm{b}}\end{array}$ & $\begin{array}{c}0.174 \pm \\
0.03 \text { bc }\end{array}$ & $\begin{array}{c}21.7 \pm \\
1.43^{\text {bcde }}\end{array}$ & $\begin{array}{l}8.33 \pm \\
0.72^{\mathrm{bc}}\end{array}$ & $\begin{array}{c}69.92 \\
\pm \\
0.72^{\mathrm{abc}}\end{array}$ & 0.98 & 1.91 & 0.49 \\
\hline $\mathrm{T} 4$ & $\begin{array}{c}16.40 \\
\pm \\
4.86^{\mathrm{de}}\end{array}$ & $\begin{array}{c}24.40 \\
\pm \\
2.28^{\mathrm{f}}\end{array}$ & $\begin{array}{c}24.00 \pm \\
1.00^{\mathrm{fgh}}\end{array}$ & $\begin{array}{c}0.04 \\
\pm 0.01^{\text {cd }}\end{array}$ & $\begin{array}{c}0.0186 \\
\pm \\
0.003^{\text {cd }}\end{array}$ & $\begin{array}{c}0.104 \\
\pm 0^{\mathrm{b}}\end{array}$ & $\begin{array}{c}0.164 \pm \\
0.01 \text { bc }\end{array}$ & $\begin{array}{l}24.5 \pm \\
4.44^{\text {bcd }}\end{array}$ & $\begin{array}{c}11.46 \\
\pm \\
1.10^{\mathrm{abc}}\end{array}$ & $\begin{array}{c}64.02 \\
\pm \\
4.27^{\mathrm{de}}\end{array}$ & 1.58 & 1.55 & 0.22 \\
\hline T5 & $\begin{array}{l}17.60 \\
\pm \underset{\text { cde }}{ } 5.28\end{array}$ & $\begin{array}{c}36.20 \\
\pm \\
1.34^{\mathrm{cd}}\end{array}$ & $\begin{array}{c}37.40 \pm \\
2.00^{\text {cd }}\end{array}$ & $\begin{array}{l}0.03 \pm \\
0.012^{\mathrm{d}}\end{array}$ & $\begin{array}{c}0.0197 \\
\pm \\
0.005^{\mathrm{c}}\end{array}$ & $\begin{array}{c}0.106 \\
\pm 0^{\mathrm{b}}\end{array}$ & $\begin{array}{c}0.154 \pm \\
0.02 \text { bc }\end{array}$ & $\begin{array}{l}18.4 \pm \\
4.97^{\text {de }}\end{array}$ & $\begin{array}{c}12.82 \\
\pm \\
3.23^{\mathrm{ab}}\end{array}$ & $\begin{array}{c}68.72 \\
\pm \\
2.94^{\text {abcd }}\end{array}$ & 2.07 & 2.13 & 0.31 \\
\hline
\end{tabular}


Table Continued..

\begin{tabular}{|c|c|c|c|c|c|c|c|c|c|c|c|c|c|}
\hline \multirow{2}{*}{ Treatments } & \multicolumn{3}{|c|}{ Cd conc. (ppm) } & \multicolumn{3}{|c|}{ Cd (mg/DBM) } & \multirow{2}{*}{$\begin{array}{c}\text { Entire } \\
\text { Plant Cd } \\
\text { Accum } \\
\text {-ulation } \\
\text { (mg/ } \\
\text { DBM) }\end{array}$} & \multicolumn{3}{|c|}{ Cd accumulation \% } & \multicolumn{2}{|c|}{$\begin{array}{c}\text { Translocation } \\
\text { Factor }\end{array}$} & \multirow{2}{*}{$\begin{array}{c}\text { Bio- } \\
\text { concentration } \\
\text { Factor }\end{array}$} \\
\hline & Roots & Stem & Leaves & $\mathbf{R}$ & $\mathbf{S}$ & $\mathbf{L}$ & & $\mathbf{R}$ & $\mathbf{S}$ & $\mathbf{L}$ & Root- Stem & $\begin{array}{l}\text { Root- } \\
\text { Leave }\end{array}$ & \\
\hline T6 & $\begin{array}{c}17.20 \\
\pm \\
1.98^{\mathrm{de}}\end{array}$ & $\begin{array}{c}38.00 \\
\pm \\
1.36^{\mathrm{bc}}\end{array}$ & $\begin{array}{l}39.40 \\
\pm 3.00^{c}\end{array}$ & $\begin{array}{l}0.03 \pm \\
0.004^{\mathrm{d}}\end{array}$ & $\begin{array}{c}0.0164 \\
\pm \\
0.004^{\mathrm{cd}}\end{array}$ & $\begin{array}{c}0.105 \\
\pm 0^{\mathrm{b}}\end{array}$ & $\begin{array}{c}0.147 \pm \\
0.03 \mathrm{bc}\end{array}$ & $\begin{array}{c}17.8 \pm \\
0.74^{\mathrm{e}}\end{array}$ & $\begin{array}{c}11.13 \\
\pm \\
0.51^{\mathrm{abc}}\end{array}$ & $\begin{array}{c}71.06 \\
\pm 0.25^{\mathrm{a}}\end{array}$ & 2.21 & 2.29 & 0.32 \\
\hline $\mathrm{T} 7$ & $\begin{array}{c}22.20 \\
\pm \\
0.38^{\mathrm{bcd}}\end{array}$ & $\begin{array}{c}27.00 \\
\pm \\
0.50^{\mathrm{ef}}\end{array}$ & $\begin{array}{c}32.00 \pm \\
4.00^{\text {de }}\end{array}$ & $\begin{array}{c}0.05 \pm \\
0.001^{\mathrm{bc}}\end{array}$ & $\begin{array}{c}0.0191 \\
\pm \\
0.002^{\mathrm{c}}\end{array}$ & $\begin{array}{c}0.137 \\
\pm 0^{\mathrm{b}}\end{array}$ & $\begin{array}{c}0.21 \pm \\
0.02^{\mathrm{b}}\end{array}$ & $\begin{array}{c}25.8 \pm \\
1.18^{\mathrm{b}}\end{array}$ & $\begin{array}{l}9.27 \pm \\
0.66^{\mathrm{abc}}\end{array}$ & $\begin{array}{c}64.97 \\
\pm \\
1.66^{\text {cde }}\end{array}$ & 1.22 & 1.44 & 0.19 \\
\hline $\mathrm{T} 8$ & $\begin{array}{c}22.00 \\
\pm \\
1.84^{\text {bcd }}\end{array}$ & $\begin{array}{c}30.60 \\
\pm \\
0.50^{\mathrm{de}}\end{array}$ & $\begin{array}{c}43.40 \pm \\
2.00^{\mathrm{bc}}\end{array}$ & $\begin{array}{c}0.03 \pm \\
0.008^{\text {cd }}\end{array}$ & $\begin{array}{c}0.0112 \\
\pm \\
0.002^{\text {cd }}\end{array}$ & $\begin{array}{c}0.118 \\
\pm 0^{\mathrm{b}}\end{array}$ & $\begin{array}{c}0.163 \pm \\
0.03^{\mathrm{bc}}\end{array}$ & $\begin{array}{c}20.9 \pm \\
0.72^{\text {bcde }}\end{array}$ & $\begin{array}{c}6.84 \pm \\
2.27^{\mathrm{c}}\end{array}$ & $\begin{array}{c}72.26 \\
\pm 1.56^{a}\end{array}$ & 1.39 & 1.98 & 0.23 \\
\hline T9 & $\begin{array}{c}25.40 \\
\pm \\
2.30^{\mathrm{abc}}\end{array}$ & $\begin{array}{l}46.00 \\
\pm \\
2.86^{\mathrm{a}}\end{array}$ & $\begin{array}{c}51.00 \\
\pm 3.00^{\mathrm{a}}\end{array}$ & $\begin{array}{c}0.02 \\
\pm 0.009 \\
\mathrm{~d}\end{array}$ & $\begin{array}{c}0.016 \\
\pm \\
0.003^{\text {cd }}\end{array}$ & $\begin{array}{c}0.086 \\
\pm 0^{\mathrm{b}}\end{array}$ & $\begin{array}{l}0.127 \pm \\
0.04^{\text {cd }}\end{array}$ & $\begin{array}{l}19.4 \pm \\
0.56^{\text {cde }}\end{array}$ & $\begin{array}{c}12.44 \\
\pm \\
0.10^{\mathrm{ab}}\end{array}$ & $\begin{array}{c}68.19 \\
\pm \\
0.66^{\text {abcd }}\end{array}$ & 1.81 & 2.01 & 0.28 \\
\hline
\end{tabular}

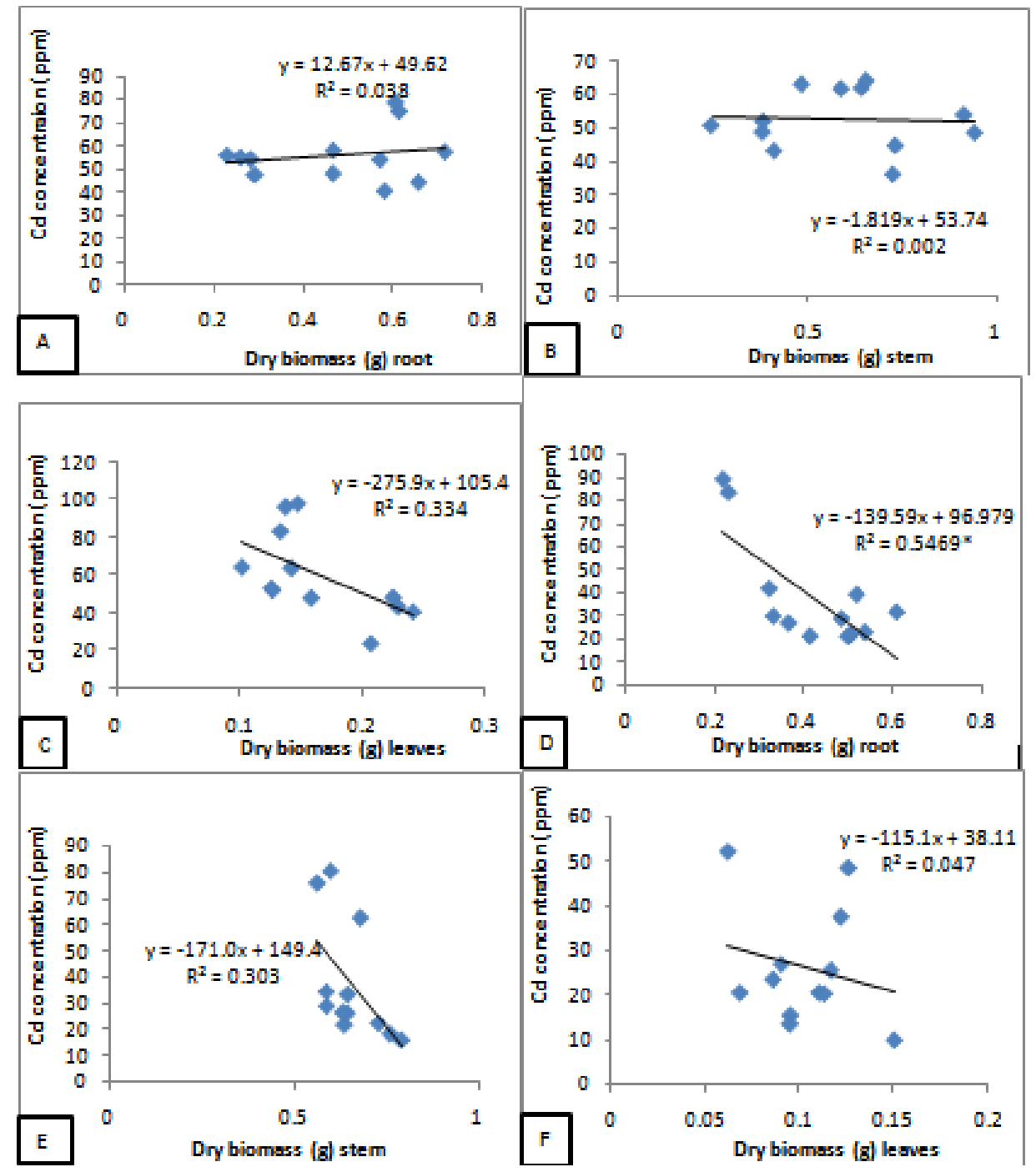

Figure 2 Correlation between dry biomass and Cd concentration within different parts of Dodonaea viscosa (A- C) and Sarcococca (D-F) plants. 
Table 5 Cadmium concentration and accumulation by various parts of Dodonaea viscosa grown in soil having different concentration s of salt and cadmium. CI, C2, C3 (50, I00, I50ppm Cd in Soil), TI, T2, T3 (I000, 3000, 6000ppm NaCl+50ppm Cd with each NaCl concentration), T4, T5, T6 (I000, 3000, 6000ppm $\mathrm{NaCl}+100 \mathrm{ppm} \mathrm{Cd}), \mathrm{T} 7, \mathrm{~T} 8, \mathrm{~T} 9(\mathrm{I000}, 3000,6000 \mathrm{ppm} \mathrm{NaCl}+150 \mathrm{ppm} \mathrm{Cd}) . \pm S D$ denote Standard deviation and different letters show the significant difference among different treatments for a specific parameter.

\begin{tabular}{|c|c|c|c|c|c|c|c|c|c|c|c|c|c|}
\hline \multirow{2}{*}{ Treatment } & \multicolumn{3}{|c|}{$\begin{array}{c}\text { Cd concentration }(\mathrm{ppm}) \\
\pm \mathrm{SD}\end{array}$} & \multicolumn{3}{|c|}{$\begin{array}{c}\text { Cd accumulation (mg/DBM) } \\
\pm S D\end{array}$} & \multirow{2}{*}{$\begin{array}{c}\text { Entire } \\
\text { plant } \\
\text { Cd (mg/ } \\
\text { DBM) } \pm \\
\text { SD }\end{array}$} & \multicolumn{3}{|c|}{ Cd accumulation \% } & \multicolumn{2}{|c|}{$\begin{array}{l}\text { Translocation } \\
\text { Factor (TF) }\end{array}$} & \multirow{2}{*}{$\begin{array}{c}\text { Bio- } \\
\text { concent } \\
\text {-ration } \\
\text { factor } \\
\text { (BF) }\end{array}$} \\
\hline & Root & Stem & Leaves & Root & Stem & Leaves & & Root & Stem & Leaf & $\begin{array}{c}\text { Root } \\
\text { to } \\
\text { Stem } \\
\end{array}$ & $\begin{array}{c}\text { Root } \\
\text { to } \\
\text { Leaves }\end{array}$ & \\
\hline $\mathrm{C} 1$ & $\begin{array}{l}57.4 \pm \\
2.02^{\mathrm{b}}\end{array}$ & $\begin{array}{l}48.8 \pm \\
1.11^{\mathrm{c}}\end{array}$ & $\begin{array}{l}41 \pm \\
2.5^{\mathrm{d}}\end{array}$ & $\begin{array}{c}0.04 \pm \\
0.0002^{\mathrm{ab}}\end{array}$ & $\begin{array}{c}0.05 \pm \\
0.0007^{\mathrm{ab}}\end{array}$ & $\begin{array}{l}0.0098 \pm \\
0.0026^{\text {bcd }}\end{array}$ & $\begin{array}{l}0.097 \pm \\
0.0031^{\mathrm{a}}\end{array}$ & $\begin{array}{c}42.5 \pm \\
1.62\end{array}$ & $\begin{array}{c}47.39 \\
\pm \\
0.85\end{array}$ & $\begin{array}{l}10.11 \\
\pm 2.46\end{array}$ & 0.85 & 0.71 & 1.02 \\
\hline $\mathrm{C} 2$ & $\begin{array}{c}74.8 \pm \\
2.86^{\mathrm{a}}\end{array}$ & $\begin{array}{c}62.2 \\
\pm \\
1.58^{\mathrm{a}}\end{array}$ & $\begin{array}{c}64.2 \pm \\
3.54^{c}\end{array}$ & $\begin{array}{c}0.05 \pm \\
0.0018^{\mathrm{a}}\end{array}$ & $\begin{array}{c}0.04 \pm \\
0.0014^{\mathrm{abc}}\end{array}$ & $\begin{array}{c}0.0091 \pm \\
0.002^{\mathrm{cd}}\end{array}$ & $\begin{array}{c}0.095 \pm \\
0.0045^{\mathrm{ab}}\end{array}$ & $\begin{array}{l}48.28 \\
\pm 1.15\end{array}$ & $\begin{array}{c}42.03 \\
\pm \\
0.82\end{array}$ & $\begin{array}{l}9.697 \\
\pm 1.79\end{array}$ & 0.83 & 0.86 & 0.68 \\
\hline $\mathrm{C} 3$ & $\begin{array}{c}78.4 \pm \\
1.36^{\mathrm{a}}\end{array}$ & $\begin{array}{c}64.4 \\
\pm 0.9^{\mathrm{a}}\end{array}$ & $\begin{array}{l}96.6 \pm \\
1.28^{\mathrm{ab}}\end{array}$ & $\begin{array}{c}0.05 \pm \\
0.0076^{\mathrm{a}}\end{array}$ & $\begin{array}{c}0.04 \pm \\
0.0063^{\mathrm{abc}}\end{array}$ & $\begin{array}{l}0.0133 \pm \\
0.0005^{\mathrm{ab}}\end{array}$ & $\begin{array}{l}0.103 \pm \\
0.0144^{\mathrm{a}}\end{array}$ & $\begin{array}{l}46.12 \\
\pm 0.7\end{array}$ & $\begin{array}{l}40.82 \\
\pm 0.3\end{array}$ & $\begin{array}{l}13.06 \\
\pm 0.98\end{array}$ & 0.82 & 1.23 & 0.49 \\
\hline $\mathrm{T} 1$ & $\begin{array}{l}44.4^{ \pm} \\
1.34^{\text {cd }}\end{array}$ & $\begin{array}{c}54.2 \\
\pm 1.8^{\mathrm{b}}\end{array}$ & $\begin{array}{c}43.8 \pm \\
0.6^{\mathrm{d}}\end{array}$ & $\begin{array}{c}0.03 \pm \\
0.0065^{c}\end{array}$ & $\begin{array}{c}0.05 \pm \\
0.0065^{\mathrm{a}}\end{array}$ & $\begin{array}{c}0.01 \pm \\
0.0001^{\text {bcd }}\end{array}$ & $\begin{array}{l}0.089 \pm \\
0.013^{\text {abc }}\end{array}$ & $\begin{array}{c}32.95 \\
\pm 0.5\end{array}$ & $\begin{array}{c}55.61 \\
\pm \\
1.16\end{array}$ & $\begin{array}{l}11.44 \\
\pm 1.61\end{array}$ & 1.22 & 0.99 & 0.99 \\
\hline $\mathrm{T} 2$ & $\begin{array}{l}54 \pm \\
1.02^{\mathrm{b}}\end{array}$ & $\begin{array}{l}62 \pm \\
1.54^{\mathrm{a}}\end{array}$ & $\begin{array}{c}48.4 \pm \\
1.6^{\mathrm{d}}\end{array}$ & $\begin{array}{c}0.03 \pm \\
0.0033^{\mathrm{bc}}\end{array}$ & $\begin{array}{c}0.04 \pm \\
0.0061^{\text {bcd }}\end{array}$ & $\begin{array}{l}0.0077 \pm \\
0.0006^{\text {cde }}\end{array}$ & $\begin{array}{l}0.075 \pm \\
0.009^{\text {bcd }}\end{array}$ & $\begin{array}{r}41.11 \\
\pm 0.43\end{array}$ & $\begin{array}{c}48.7 \\
\pm \\
1.29\end{array}$ & $\begin{array}{l}10.19 \\
\pm 1.69\end{array}$ & 1.15 & 0.9 & 1.14 \\
\hline T3 & $\begin{array}{l}58 \pm \\
0.9^{\mathrm{b}}\end{array}$ & $\begin{array}{c}63.4 \\
\pm \\
1.58^{a}\end{array}$ & $\begin{array}{c}98.2 \pm \\
1.56^{\mathrm{a}}\end{array}$ & $\begin{array}{c}0.03 \pm \\
0.0029^{c}\end{array}$ & $\begin{array}{c}0.03 \pm \\
0.0022^{\text {cde }}\end{array}$ & $\begin{array}{c}0.0145 \pm \\
0.0009^{\mathrm{a}}\end{array}$ & $\begin{array}{l}0.072 \pm \\
0.006^{\mathrm{cd}}\end{array}$ & $\begin{array}{c}37.4 \pm \\
0.65\end{array}$ & $\begin{array}{c}42.34 \\
\pm \\
1.05\end{array}$ & $\begin{array}{l}20.27 \\
\pm 0.43\end{array}$ & 1.09 & 1.69 & 1.32 \\
\hline $\mathrm{T} 4$ & $\begin{array}{c}40.6 \pm \\
0.58^{\mathrm{d}}\end{array}$ & $\begin{array}{c}45 \pm \\
0.86^{d}\end{array}$ & $\begin{array}{c}48.6 \pm \\
1.8^{\mathrm{d}}\end{array}$ & $\begin{array}{c}0.02 \pm \\
0.0033^{\text {cd }}\end{array}$ & $\begin{array}{c}0.03 \pm \\
0.0047^{\mathrm{cd}}\end{array}$ & $\begin{array}{l}0.0109 \pm \\
0.0024^{\mathrm{abc}}\end{array}$ & $\begin{array}{c}0.067 \pm \\
0.0057^{\text {cde }}\end{array}$ & $\begin{array}{l}35.02 \\
\pm 1.63\end{array}$ & $\begin{array}{c}48.9 \\
\pm \\
3.28\end{array}$ & $\begin{array}{l}16.08 \\
\pm 4.90\end{array}$ & 1.11 & 1.2 & 0.44 \\
\hline T5 & $\begin{array}{c}47.6 \pm \\
0.86^{c}\end{array}$ & $\begin{array}{l}49 \pm \\
1.02^{c}\end{array}$ & $\begin{array}{c}53.6 \pm \\
3^{\text {cd }}\end{array}$ & $\begin{array}{c}0.01 \pm \\
0.0016 \\
\text { de }\end{array}$ & $\begin{array}{c}0.02 \pm \\
0.0023^{\mathrm{fg}}\end{array}$ & $\begin{array}{l}0.0068 \pm \\
0.0024^{\text {de }}\end{array}$ & $\begin{array}{l}0.039 \pm \\
0.0024^{\mathrm{fg}}\end{array}$ & $\begin{array}{c}35.3 \pm \\
1.45\end{array}$ & $\begin{array}{l}47.55 \\
\pm 4.6\end{array}$ & $\begin{array}{l}17.15 \\
\pm 3.19\end{array}$ & 1.03 & 1.13 & 0.49 \\
\hline T6 & $\begin{array}{c}54.2 \pm \\
2.86^{\mathrm{b}}\end{array}$ & $\begin{array}{c}52.2 \\
\pm \\
1.04^{\mathrm{bc}}\end{array}$ & $\begin{array}{c}83.6 \pm \\
1.34^{\mathrm{b}}\end{array}$ & $\begin{array}{c}0.02 \pm \\
0.0008^{\text {de }}\end{array}$ & $\begin{array}{c}0.02 \pm \\
0.0008^{\mathrm{efg}}\end{array}$ & $\begin{array}{l}0.0111^{ \pm} \\
0.0011^{\mathrm{abc}}\end{array}$ & $\begin{array}{c}0.046 \pm \\
0.0014^{\mathrm{efg}}\end{array}$ & $\begin{array}{r}33.04 \\
\pm 0.78\end{array}$ & $\begin{array}{c}43.09 \\
\pm \\
2.81\end{array}$ & $\begin{array}{l}23.87 \\
\pm 2.52\end{array}$ & 0.96 & 1.54 & 0.58 \\
\hline $\mathrm{T} 7$ & $\begin{array}{c}48.2 \pm \\
1.58^{\mathrm{c}}\end{array}$ & $\begin{array}{c}36.4 \\
\pm \\
1.18^{\mathrm{e}}\end{array}$ & $\begin{array}{c}24.2^{ \pm} \\
14.3^{\mathrm{e}}\end{array}$ & $\begin{array}{c}0.02 \pm \\
0.0006 \\
\text { cde }\end{array}$ & $\begin{array}{c}0.03 \pm \\
0.0005^{\text {def }}\end{array}$ & $\begin{array}{l}0.005 \pm \\
0.0018^{\mathrm{e}}\end{array}$ & $\begin{array}{c}0.054 \pm \\
0.0028^{\text {def }}\end{array}$ & $\begin{array}{c}41.7 \pm \\
0.89\end{array}$ & $\begin{array}{c}48.86 \\
\pm \\
1.55\end{array}$ & $\begin{array}{c}9.44 \pm \\
2.34\end{array}$ & 0.76 & 0.5 & 0.26 \\
\hline $\mathrm{T} 8$ & $\begin{array}{l}55 \pm \\
1.84^{\mathrm{b}}\end{array}$ & $\begin{array}{c}43.4 \\
\pm \\
0.92^{\mathrm{d}}\end{array}$ & $\begin{array}{c}52.4 \pm \\
1.2^{\mathrm{cd}}\end{array}$ & $\begin{array}{c}0.01 \pm \\
0.0041^{\text {de }}\end{array}$ & $\begin{array}{c}0.02 \pm \\
0.0049^{\mathrm{fg}}\end{array}$ & $\begin{array}{l}0.0067 \pm \\
0.0002^{\text {de }}\end{array}$ & $\begin{array}{l}0.039 \pm \\
0.0091^{\mathrm{fg}}\end{array}$ & $\begin{array}{c}36.77 \\
\pm 0.7\end{array}$ & $\begin{array}{c}46.13 \\
\pm \\
0.86\end{array}$ & $\begin{array}{c}17.1 \pm \\
1.48\end{array}$ & 0.79 & 0.95 & 0.32 \\
\hline T9 & $\begin{array}{c}55.8 \pm \\
1.3^{\mathrm{b}}\end{array}$ & $\begin{array}{l}51 \pm \\
1.4^{\mathrm{bc}}\end{array}$ & $\begin{array}{c}64.6 \pm \\
1.22^{\mathrm{c}}\end{array}$ & $\begin{array}{c}0.01 \pm \\
0.0013^{\mathrm{e}}\end{array}$ & $\begin{array}{c}0.01 \pm \\
0.0026^{g}\end{array}$ & $\begin{array}{l}0.0066 \pm \\
0.0013^{\text {de }}\end{array}$ & $\begin{array}{l}0.032 \pm \\
0.0009^{g}\end{array}$ & $\begin{array}{r}40.05 \\
\pm 3.34\end{array}$ & $\begin{array}{c}39.35 \\
\pm \\
6.79\end{array}$ & $\begin{array}{c}20.6 \pm \\
3.45\end{array}$ & 0.91 & 1.16 & 0.37 \\
\hline
\end{tabular}

\section{Discussion}

Cadmium contaminated soil decreases the plant growth, and it could be the negative effect of cadmium on uptake of nutrient and their distribution in the plant cells. ${ }^{21}$ Its accumulation in plants cell may negatively affect the growth and development of a plant by causing a decrease in the enzymatic activities ${ }^{22}$ and $^{23}$ disturb respiration, photosynthesis, ${ }^{24}$ stomatal closure $^{25}$ and reduction of nutrient uptake ${ }^{26}$ The present result showed a decrease in plant growth and biomass due to $\mathrm{Cd}$ toxicity. Similar effects of $\mathrm{Cd}$ were reported by various investigators on different plants such as on Cucumus sativus ${ }^{27}$ Lemna polyrrhiza ${ }^{28}$ and on Glycyrrhiza uralensis. ${ }^{29}$ Cadmium may affect the root elongation by reducing water and nutrient absorption, decreasing the transpiration rate and consequently decreasing growth rate. ${ }^{\mathrm{s}}$

Salinity in soil and water produces stress condition for plants and may lead to reduction in growth and biomass of a plant. ${ }^{31}$ It affects plant in three ways, i.e. by decreasing its water potential, ionic imbalance or disturbances in ion homeostasis and its toxicity. Salinity cause physiological drought condition in plants and causes both osmotic as well as ionic stress, thus induce a reduction in growth. ${ }^{32}$ The suppression of growth is directly related to the total concentration of soluble salts. ${ }^{33}$ In current experiment salt $(\mathrm{NaCl})$ showed an increasing effect on $\mathrm{Cd}$ absorption and accumulation within plant tissues. This increase in $\mathrm{Cd}$ content of plant might be due to two mechanismsi.e. exchange of metals from sorption sites in soil by the cationic component and formation of stable metal complexes with the chloride anion. ${ }^{34}$ Addition of $\mathrm{NaCl}$ increased $\mathrm{Cd}$ concentration in the soil solution and accumulation in the leaf of Swiss chard. ${ }^{35}$ It demonstrate that bioavailability of $\mathrm{Cd}$ is enhanced under saline conditions. Human-induced salinization and trace element contamination are widespread and increasing rapidly. Phytoaccumulation, as the crucial entry pathway for bio-toxic $\mathrm{Cd}$ into the human food stuffs, correlates positively with rhizosphere salinity. Organic matter decreases the bioavailable $\mathrm{Cd} 2+$ pool and therefore restricts its Phytoextraction. Sodium salt $(\mathrm{NaCl})$ showed reduction in plant growth and biomass compared to other treatments which might be due to its negative effect on production of endogenous plant growth regulators. ${ }^{36}$ 
In the present result, $\mathrm{Cd}$ significantly reduced the plant growth, total water content (TWC) and biomass, the same result have been presented by Rubio et al., ${ }^{37}$ who reported that plant growth was reduced by $\mathrm{Cd}$ uptake and its distribution within cells. According to, ${ }^{38} \mathrm{Cd}$ affects plant growth by damaging membrane permeability and elongation of cell. . Current result showed that $\mathrm{NaCl}$ increased uptake of $\mathrm{Cd}$ at low concentration up to certain level while maximum amount of $\mathrm{NaCl}$ salt did not increase the Cd Phytoextraction. Similar results were found in the work of, ${ }^{30}$ where sodium salt enhanced Phytoextraction of $\mathrm{Cd}$ in optimum condition and cause toxicity to plants that ultimately affected the growth parameter.

In this result specially in hydroponic condition growth parameter were reduced gradually with increase of sodium salt, because salt enhanced the translocation factor of $\mathrm{Cd}^{39}$ stated that sodium chloride is a biological dilution and improved the $\mathrm{Cd}$ concentration with increasing sodium salt concentration. ${ }^{40}$ suggest that increasing salinity increases cadmium uptake and the reduction of growth has direct proportion to the sodium salt concentration. ${ }^{41}$ Salt $(\mathrm{NaCl})$ addition to growth media showed an increasing effect on the $\mathrm{Cd}$ concentration in different parts of the plant. Cd concentration was enhanced by the gradual increase of salinity. ${ }^{42}$ Salinity enhanced the chloro-complex with $\mathrm{Cd}$ which may lead to increase the translocation of $\mathrm{Cd}$ in the cell. ${ }^{42}$ A Similar increase in Cd concentration in relation with the increase in the $\mathrm{NaCl}$ concentration in soil has been reported in potato and sunflower. ${ }^{43}$

The Cd accumulation increased with the increase of salinity and maximum concentrations were reported in plant roots due to $\mathrm{Cd}$ elevation through salt. ${ }^{44}$ These results are in general agreement with previous studies in which Tamarix ramosissima showed a marked diminution in growth in response to salinity but no diminution in photosynthesis over a salinity gradient from 0 to $200 \mathrm{mM} \mathrm{NaCl}$ and it was concluded that growth was negatively affected by salinity due to diversion of energy for increased respiration and salt pumping. ${ }^{45}$ Phyto remediation is a right choice which is applicable to multicontamination. Laboratory and field trials have proven successful, but this ideal technique is in all cases dependent on plant growth ability on low-fertility soil. While contaminant concentration has often been proposed as an explanation for plant growth limitation, other factors, commonly occurring in industrial soils, such as salinity, should be considered. In order to achieve the goal, the accumulation of $\mathrm{Cd}$ via root uptake at different saline conditions were investigated as there is notable evidence that salinity is a key factor in the translocation of metals from roots to the aerial parts of the plant. ${ }^{46}$

\section{Conclusion}

Dodoneae plants grown in soil as well as in acidic and sodic soil too but show tolerance and were found as Cd hyper accumulators, while Hemerocallis plants was not hyper accumulators of $\mathrm{Cd}$. The salt of sodium, although, increased the cadmium concentration in the plant tissues but showed negative effect on plant growth and biomass. Increasing the sodium salt concentration decreased biomass of the plants but showed an increasing effect on the Cd uptake and concentration within different parts of plant. From the results it is clear that the use of saline soil/water containing cadmium metal should be avoided to use for agricultural purposes because of higher absorption of $\mathrm{Cd}$ by plants in saline soil/water. It is strongly recommended that plantation and cultivation of Dodoneae plant is very important for phyto extraction of metals in saline soil and conservation of barren rocks.

\section{Acknowledgements}

The higher education commission of Pakistan (HEC) is highly acknowledged for funding this project under the scheme of indigenous PhD fellowship programme of HEC 2012 to 2014.

\section{Conflict of interest}

The author declares no conflict of interest.

\section{References}

1. Singh RP, Dhania G, Sharma A, et al. Bio-technological approaches to improve phytoremediation efficiency for environments contaminants. In: Environmental bioremediation technologies. Environmental Bioremediation Technologies. 2007. p. 223-258.

2. Sima NAKK, Askari H, Mirzaei HH, et al. Genotypic differential responses of three forage species to calcium supplement in saline conditions. J Plant Nutr. 2009;32(4):579-597.

3. Jaleel CA, Manivannan P, Kishorekumar A, et al. Alterations in osmoregulation, antioxidant enzymes and indole alkaloid levels in Catharanthus roseusexposed to water deficit. Colloids and Surfaces B. Biointerfaces. 2007;59(2):150-157.

4. Bauddh K, Singh RP. Growth, tolerance efficiency and phytoremediation potential of Ricinus communis(L.) and Brassica juncea (L.) in salinity and drought affected cadmium contaminated soil. Ecotox Environ Safety. 2012;85:13-22.

5. Flowers TJ, Hajiabagheri MA. Salinity tolerance in Hordeum vulgare:ion concentration on root cells of cultivars differing in salt tolerance. Plant soil. 2001;231(1):1-9.

6. Bhaskar P, Bauddh K, Singh RP. Differential response of two high yielding cultivars of Indian mustard (Brassica juncea L.) to $\mathrm{NaCl}$ salinity during seed germination and early seedling growth. J Ecophysiol Occup Hlth. 2009;9:137-144.

7. Kaya M, Kaya G, Kaya MD, et al. Interaction between seed size and $\mathrm{NaCl}$ on germination and early seedling growth of some Turkish cultivars of chickpea (Cicer arietinum L.). J Zhejiang Univ Sci B. 2008;9(5):371-377.

8. Epstein E, Norlyn JD, Rush DW, et al. Saline culture of crops:a genetic approach. Science. 1980;210(4468):399-404.

9. Hart JJ, Welch RM, Norvell WA, et al. Characterization of cadmium binding, uptake and translocation in intact seedlings of bread and durum wheat cultivars. Plant Physiol. 1998;166(4):1413-1420.

10. Oliveira JA, Oliva MA, Cambraia J, et al. Absorption, accumulation and distribution of cadmium by to soybean CVS. R Bras Fisiol Veg. 1999;6:91-95.

11. Salt DE, Rauser WE. MgA TP-dependent transport of phytochelatins across the tonoplast of oat roots. Plant Physio. 1995;107(4):1293-1301.

12. Arora M, Kiran B, Rani S, et al. Heavy metal accumulation in vegetables irrigated with water from different sources. $J$ of Food Chem. 2008;111(4):811-815.

13. Zhu JK, Hasegawa RM, Bressan RA. Molecular aspects of osmotic stress. Crit Rev Plant Sci. 1997;16(3):253-277.

14. Laidlaw WS, Baker AJM, Gregory D, et al. Phytoremediation of bio solids and soils contaminated with heavy metals at the Western Treatment Plant, Research and Technology Division, Melbourne Water cooperation; 2007.

15. Ashraf M, Harris PJC. Potential biochemical indicators of salinity tolerance in plants. Plant Sci. 2004;166(1):3-16. 
16. McWilliam JR. The national and international importance of drough and salinity effects on agricultural production. Austral J Plant Physiol. 1986;13:1-13.

17. Ghassemi F, Jakeman AJ, Nix HA. Salinization of Land and Water Resources. Canberra, Australia: University of New South Wales Press Ltd; 1995.

18. Mashali AM. FAO global network in soil Mc William JR1 986, Ghassemi F 1995, management for sustainable use of salt affected soils, International Workshop; 1999. p. 1-30.

19. Menemen, Evelin H, Kapoor R, et al. Arbuscular mycorrhizal Fungi in alleviation of salt stress:a review. Ann Bot. 2009;104(7):1263-1281.

20. Allen SE, Grimshaw AH, Parkinson JA, et al. Chemical Analysis of Ecological Materials. UK: Blackwell Scientific Publications; 1974.

21. Hernandez LE, Carpena-Ruiz R, Garate A. Alterations in the mineral nutrition of pea seedlings exposed to cadmium. J of Plant Nutrition and Soil Sci. 1996;19(12):1581-1598.

22. Ouarili O, Boussama N, Zarrouk M, et al. Cadmium and copper-induced changes in tomato membrane lipids. Phytochemistry. 1997;45(7):1343350 .

23. Van Assche F, Clijsters H. Effects of metals on enzyme activity in plants. Plant Cell Env. 1990;13(3):195-206.

24. Vassilev A, Lidon F, Matos MDC, et al. Photosynthetic performance and content of some nutrients in cadmium- and copper-treated barley plants. J of Plant Nut. 2002;25(11):2343-60.

25. Barcelo J, Poschenrieder C. Plant water relations as affected by heavy metal stress:a review. J of Plant Nut. 1990;13(1):1-37.

26. Sanità di Toppi L, Gabbrielli R. Response to cadmium in higher plant. Environ Exp Bot. 1999;41(2):105-130.

27. Abu-Muriefah SS. Growth parameters and elemental status of cucumber (Cucumus sativus) seedlings in response to cadmium accumulation. Int J Agri Biol. 2008;10:261-266.

28. John R, Ahmad P, Gadgil K, et al. Effect of cadmium and lead on growth, biochemical parameters and uptake in Lemna polyrrhiza L. Plant Soil Env. 2008;54(6):262-270.

29. Zheng G, Lv HP, Gao S, et al. Effects of cadmium on growth and Antioxidant responses in Glycyrrhiz auralensis seedlings. Plant Soil Env. 2010;56(11):508-515.

30. Chen ZS, Lee GJ, Liu JC. The effects of chemical remediation treatments on the extractability and speciation of cadmium and lead in contaminated soils. Chemosphere. 2000;41(1-2):235-242.

31. Sirguey C, Ouvrard S. Contaminated soils salinity, a threat for phytoextraction. Chemosphere. 2013;91(3):269-274.

32. Hayashi H, Murata N. Genetically engineered enhancement of salt tolerance in higher plants, Molecular Mechanisms and Molecular Regulation. Elsevier Amsterdam; 1998. p. 133-148.
33. Flowers TJ, Troke PF, Yeo AR. The mechanisms of salt tolerance in halophytes. Annual Review of Plant Physio. 1997;28:89-121.

34. Schmidt U. Enhancing phytoextraction:The effect of chemical soil manipulation on mobility, plant accumulation and leaching of heavy metals. J Envi Qual. 2003;32(6):1939-1954.

35. Smolders E, Lambregts RM, Mclaughlin MJ, et al. Effect of soil solution chloride on Cd availability to swiss chard. J Env Qual. 1998;27(2):426431

36. El-Khallal SM, Hathout TA, Abd-El-Raheim A, et al. Brassinolide and salicylic acid induced growth, biochemical activities and productivity of maize plants grown under salt stress. Rese Jof Agri Biolog Sci. 2009;5(4):380-390

37. Rubio MI, Escrig I, Martínez-Cortina C, et al. Cadmium And nickel accumulation in rice plants: effects on mineral nutrition and possible interactions Of abscisic and gibberellic acids. Plant Growth Regulation 1994;14(2):151-157.

38. Mousa Khatamipour, Eissa Piri, Yaser Esmaeilian, et al. Toxic effect of cadmium on germination, seedling growth and proline content of Milk thistle (Silybummarianum). Ann Biol. 2011;Res2(5):527-532.

39. Chai MW, Shi FC, Li RL, et al. Effect of $\mathrm{NaCl}$ on growth and $\mathrm{Cd}$ accumulation of halophyte Spartinaalterniflora under $\mathrm{CdCl} 2$ stress. South African J of Bot. 2013;85:63-69.

40. Manousaki E, Kalogerakis N. Phytoextraction of $\mathrm{Pb}$ and $\mathrm{Cd}$ by the MediterraneanSalt bush (Atriplex halimus L.): metal uptake in relation to salinity. IJ Env Sci poll Res. 2009;16(7):844-854.

41. Greenway H, Munns R. Mechanism of salt tolerance in non-halophytes Ann Rev of Plan Physiol. 1999;3:149-190.

42. Helal HM, Upenov A, Issa GJ. Growth and uptake of $\mathrm{Cd}$ and $\mathrm{Zn}$ by Leucaena leucocephala in reclaimed soils as affected by $\mathrm{NaCl}$ salinity. $J$ of Plan Nutr Soil Sci. 1999;162:589-592.

43. McLaughlin, MJ, Tiller, KG, Beech TA, et al. Soil salinity causes elevated cadmium concentrations in field-growth potato tubers. J Env Sci. 1994;23L:1013-1018.

44. Pedro CA, Santos MSS, Ferreira SMF, et al. The influence of cadmium contamination and salinity on the survival, growth and phytoremediation capacity of the saltmarsh plant Salicornia ramosissima. J of Marine Env Res. 2013;92:197-205.

45. Gabrijel O, Davor R, Zed R, et al. Cadmium accumulation by muskmelon under salt stress in contaminated organic soil. Sci of total env. 2009;407(7):2175-2182

46. Otte ML. Contamination of coastal wetlands with heavy metals:factors affecting uptake of heavy metals by salt marsh plants, In: Rozema J, et al. editors. Ecological Responses to Environmental Stresses. USA: Kluwer Academic; 1991. 